\title{
Misalignments calibration in small-animal PET scanners based on rotating planar detectors and parallel-beam geometry
}

\author{
M Abella ${ }^{1,6}$, E Vicente $^{2,3,6}$, A Rodríguez-Ruano ${ }^{4}$, S España $^{2,7}$,

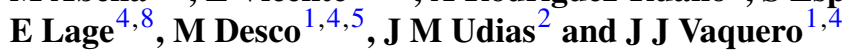 \\ ${ }^{1}$ Departamento de Bioingeniería e Ingeniería Aeroespacial, Universidad Carlos III de Madrid, \\ Madrid, Spain \\ ${ }^{2}$ Grupo de Física Nuclear, Dpto. Física Atómica, Molecular y Nuclear, Universidad Complutense \\ de Madrid, Madrid, Spain \\ ${ }^{3}$ Instituto de Estructura de la Materia, Consejo Superior de Investigaciones Científicas (CSIC), \\ Madrid, Spain \\ ${ }^{4}$ Instituto de Investigación Sanitaria Gregorio Marañón (IiSGM), Madrid, Spain \\ ${ }^{5}$ Centro de Investigación en Red de Salud Mental (CIBERSAM, CIBER CB07/09/0031), \\ 28007 Madrid, Spain \\ E-mail: monica.abella@ing.uc3m.es and esther@nuclear.fis.ucm.es
}

\begin{abstract}
Technological advances have improved the assembly process of PET detectors, resulting in quite small mechanical tolerances. However, in high-spatialresolution systems, even submillimetric misalignments of the detectors may lead to a notable degradation of image resolution and artifacts. Therefore, the exact characterization of misalignments is critical for optimum reconstruction quality in such systems. This subject has been widely studied for CT and SPECT scanners based on cone beam geometry, but this is not the case for PET tomographs based on rotating planar detectors. The purpose of this work is to analyze misalignment effects in these systems and to propose a robust and easy-to-implement protocol for geometric characterization. The result of the proposed calibration method, which requires no more than a simple calibration phantom, can then be used to generate a correct 3D-sinogram from the acquired list mode data.
\end{abstract}

\footnotetext{
6 These authors contributed equally to this work.

7 Present address: Department of Electronics and Information Systems, MEDISIP, Ghent University-IBBT-IBiTech, B-9000 Ghent, Belgium.

8 Present address: Madrid-MIT M-Visión Consortium, Research Laboratory of Electronics, Massachusetts Institute of Technology, Cambridge, USA.
} 


\section{Introduction and motivation}

The increasing number of animal models of human diseases used in translational research makes small-animal imaging an essential tool to assess biological functions. This approach makes it possible to carry out new types of studies, such as the monitoring of transgenic expression in genetically modified mice or in vivo monitoring of tumor response to therapy (Pomper 2001, Lewis and Achilefu 2002, Massoud and Gambhir 2003). In these systems, the dimensions of the structures whose biochemical functions we want to analyze impose additional system requirements not shared by clinical scanners (Lecomte 2004). To address the same biological questions that are already being investigated in humans, both systems must provide a similar relation between spatial resolution and the size of the organ under study, which is known as 'image equivalence' (Jagoda et al 2004). This suggests that a small-animal system would have to reconstruct images with a resolution below $1 \mathrm{~mm}$ in all directions, whereas systems for imaging human tissue typically have a resolution of $\sim 4-10 \mathrm{~mm}$. Therefore, exact geometric characterization is critical in small-animal scanners, as even submillimetric misalignments of the detectors may result in noticably degraded image resolution and artifacts.

Methods for estimating geometrical parameters of tomography scanners have been investigated since 1987, mainly for CT and SPECT systems based on fan-beam geometry (Gullberg et al 1987, Hsieh 1992) and cone-beam geometry (Gullberg et al 1990, Noo et al 2000, Beque et al 2003, Sun et al 2007). Systems based on parallel-beam geometry have received little attention. Azevedo et al (1990) studied methods for estimating the center of rotation (COR) in CT using sinogram data; Busemann-Sokole (1987) proposed a method for the determination of collimator hole angulations in SPECT scanners and, more recently, Donath et al (2006) presented an iterative procedure for the determination of the position of the optimum COR intended for any tomography system. Few contributions have been made on the specific case of rotating PET systems, except for a preliminary study of the effect of misalignments by our group (Abella et al 2006). More recently, Pierce et al (2009) proposed a new calibration method to determine block positions in a system based on a ring of PET detectors. To the best of our knowledge, there are no systematic studies of the effect of all possible misalignments in a tomography system based on parallel-beam geometry with rotating planar detectors.

Calibration techniques are usually based on analytical expressions applicable to the acquisition of ad hoc phantoms, which sometimes require precise positioning in the field of view (FOV). In our experience, these analytic algorithms should only be used to provide an initial appraisal of misalignment parameters, since most of them make assumptions of ideal or negligible conditions that may not hold in real scanners, and usually require further fine tuning.

We analyzed misalignment effects in PET systems based on rotating detectors with parallel-beam geometry and defined a robust and easy-to-implement protocol for geometric characterization. The result of the calibration step can then be used to generate the correct 3D-sinogram from the acquired list mode data. Section 2 presents a description of the simulations used. Section 3 describes the misalignments considered in this work and provides a theoretical study of the effects of these misalignments on projection data and of the tolerance of the system to each one, in order to define an easy way for detection and calibration. Finally, section 4 proposes an algorithm to measure these misalignments in a real scanner. 

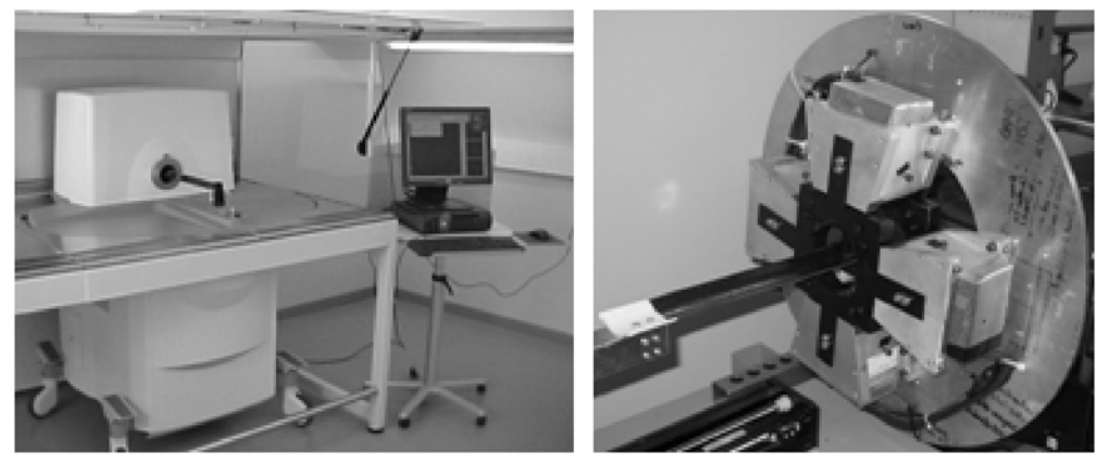

Figure 1. rPET scanner. Left panel shows a real experimental set-up. Right panel shows the first scanner prototype with the covers removed.

\section{Monte Carlo simulations using PeneloPET}

In order to study and quantify the effects of the different geometrical misalignments and to evaluate the proposed algorithm, we used the simulation package PeneloPET (España et al 2009), an easy-to-use application, which allows comprehensive simulations of PET systems within PENELOPE (Baró et al 1995). PeneloPET simulates PET systems based on crystal array blocks coupled to photodetectors and allows the user to define radioactive sources, detectors, shielding and other parts of the scanner. Detector blocks can be displaced from their regular positions in order to build non-regular complex geometries or to introduce misalignments of detectors.

Simulations were based on the high-resolution animal rPET scanner (SEDECAL Medical Systems, Spain) (Vaquero et al 2004, Vaquero et al 2005). This system has four detectors arranged as two orthogonal pairs attached to a rotating gantry (figure 1). Each detector comprises an array of $30 \times 30$ mixed lutetium silicate (MLS) crystals measuring $1.5 \mathrm{~mm} \times 1.5 \mathrm{~mm} \times 12 \mathrm{~mm}$ in size, with $0.1 \mathrm{~mm}$ of reflective material between the crystals, and is optically coupled to a Hamamatsu H8500 flat-panel position-sensitive photomultiplier (PS-PMT). The PS-PMT can resolve a matrix of $28 \times 28$ crystals from the original $30 \times 30$ array (Vaquero et al 2005).

The system gantry rotates $180^{\circ}$ covering a total angle of $194^{\circ}$ due to the solid angle subtended by the detectors. Each pair of detectors measures photon coincidences generated in a cylindrical FOV of $44.8 \mathrm{~mm}$ (diameter) $\times 44.8 \mathrm{~mm}$ (height).

Data measured by each detector pair are rearranged into a set of direct 2D sinograms, which only include lines of response (LOR) from transaxial slices, and oblique 2D sinograms, which traverse several slices, thus conforming a $3 \mathrm{D}$ sinogram as a function of three variables $(\rho, \theta, s) . \rho$ is the distance between the LOR and the COR, ranging from 0 to $R$ (radius of the FOV), $\theta$ is the azimuthal angle between the LOR and the $Y_{0}$-axis, ranging from $0^{\circ}$ to $180^{\circ}$ (figure 2, left) and $s$ is an integer variable which accounts for the different 2D sinograms, ranging from 0 to $28^{2}$. These sinograms cover all the possible LORs registered by the detector elements on different rows.

Data for angles over $360^{\circ}$ are reallocated at the corresponding $2 \mathrm{D}$ sinogram position in the range $0^{\circ}-180^{\circ}$, as derived from the symmetry property

$$
p(\rho, \theta+\pi)=p(-\rho, \theta)
$$




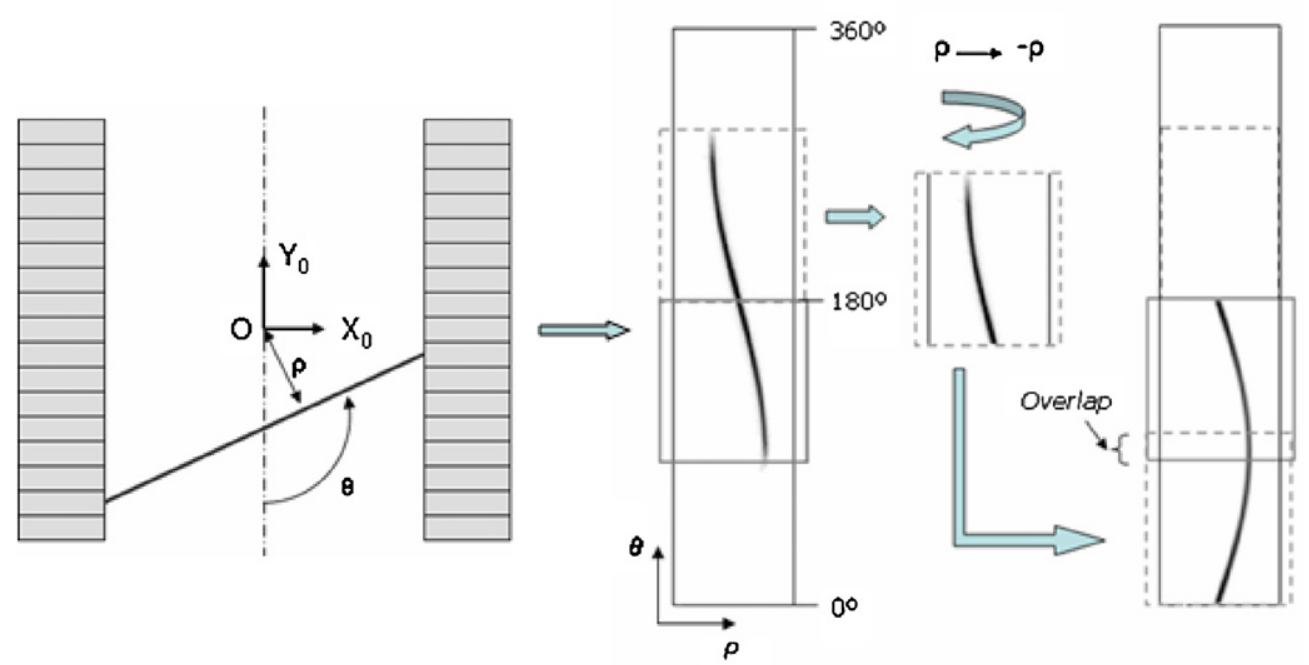

Figure 2. Left: transaxial view showing the 2D sinogram variables $\rho$ and $\theta$ used to parameterize the LORs. Right: rearrangement of the data, from a $360^{\circ} 2 \mathrm{D}$ sinogram into a $180^{\circ} 2 \mathrm{D}$ sinogram.

where $p(\rho, \theta)$ is the data measured in an LOR defined by the pair $(\rho, \theta)$ for a given $s$. As a result, there is a region where the extra $14^{\circ}$ from the first and last positions of the detectors overlap (figure 2, right). Each 2D sinogram has 55 radial and 120 angular bins, resulting in a spacing of $0.8 \mathrm{~mm}$ and $1.5^{\circ}$ in the radial and angular directions, respectively.

Two point source studies with different misalignment configurations were simulated: one located at the center of the FOV and the other one at $10 \mathrm{~mm}$ from the center of the FOV, both in the central axial slice. The point source consisted of a sphere $(0.5 \mathrm{~mm}$ of diameter $)$ with 1.1 $\mathrm{MBq}(30 \mu \mathrm{Ci})$ of ${ }^{18} \mathrm{~F}$ in a cubic phantom $\left(1 \times 1 \times 1 \mathrm{~cm}^{3}\right)$ of water, resembling a real point source phantom for calibration. In order to have enough SNR, we chose an acquisition time of $320 \mathrm{~s}$, which provided a total number of acquired coincidences of around $7 \times 10^{6}$ (center) and $6 \times 10^{6}(1 \mathrm{~cm}$ off-center $)$.

Finally, a Hot Derenzo Phantom acquisition was simulated with and without misalignments to evaluate the effect of the different calibration outputs with the proposed method on the image quality. The initial activity was $11.2 \mathrm{MBq}(300 \mu \mathrm{Ci})$ and the acquisition time $560 \mathrm{~s}$ (total number of acquired coincidences: $3 \times 10^{7}$ ).

\section{Study of the effect of misalignments}

This section presents a study of the effect of different geometrical misalignments on a detector pair: linear shifts of the detectors ( $x, y$ and $z$ in figure 3, right) and angles between the detector and the gantry ( $\xi$, skew; $\sigma$, tilt; $\gamma$, slant in figure 3 , left).

For each type of misalignment, we study its effect on the positioning of the LORs, the 3D sinogram and the reconstructed image. We also calculate the tolerance of the system for each case. These effects can be classified according to the schema shown in figure 4.

\subsection{Misalignment along the y-axis}

In a rotating PET scanner, a misalignment along the $y$-axis, hereafter referred to as ' $y$-offset', produces two types of effects. The first one ('asymmetrical component') derives 

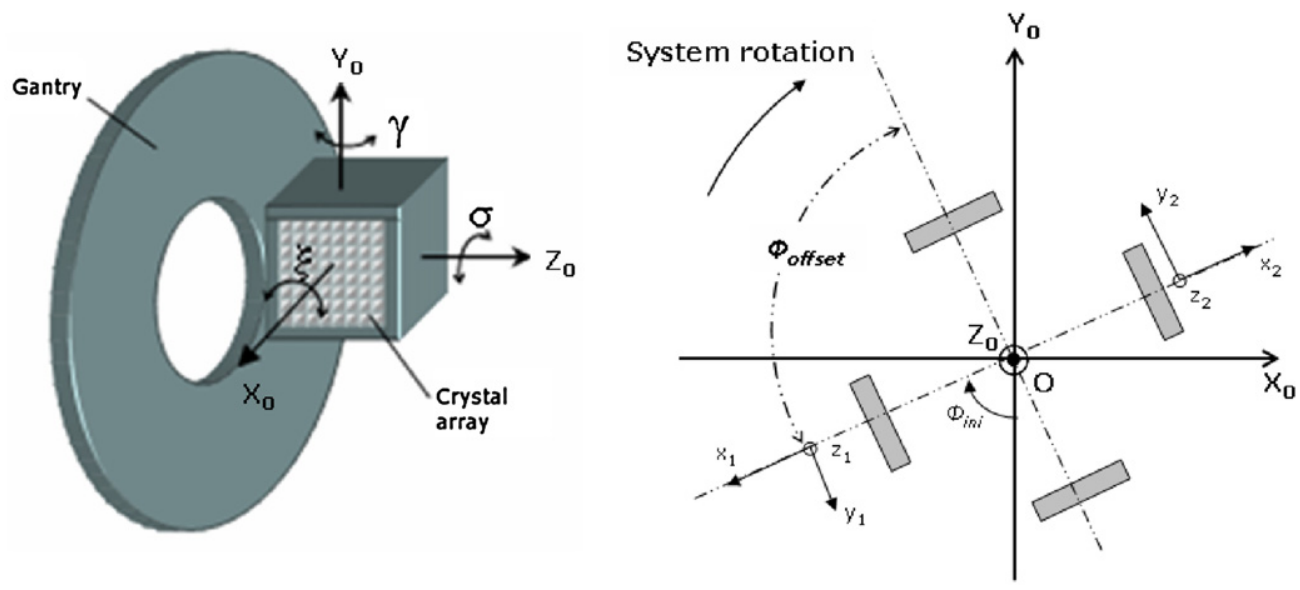

Figure 3. Left: detector attached to the rotating gantry showing the three possible sources of angular errors in the detector position in relation to the gantry: $\sigma, \gamma$ and $\xi$. Right: axial view of the system showing the coordinate systems used: $X_{0}, Y_{0}$ and $Z_{0}$ are absolute coordinates and $\left(x_{1}, y_{1}\right.$, $\left.z_{2}\right)$ and $\left(x_{2}, y_{2}, z_{2}\right)$ are coordinates relative to detectors 1 and 2 , respectively.

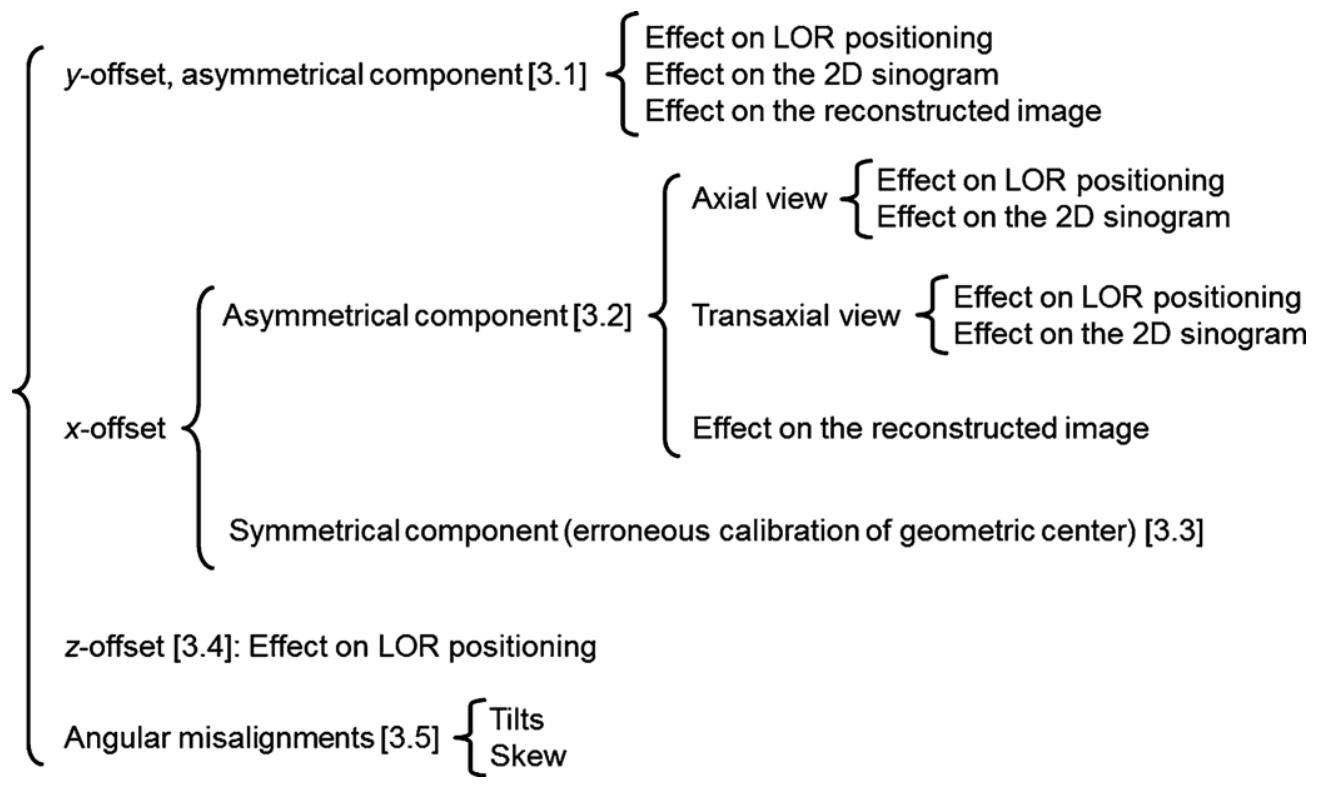

Figure 4. Classification of the effects considered. Numbers in brackets indicate the subsection where that effect is analyzed.

from a differential misalignment between opposite detectors and leads to a mismatch between the geometrical center and the COR. The second effect ('symmetrical component') produces an error in the $\theta$ parameter when positioning the LORs, although this will only result in a loss of resolution. This section describes the asymmetrical component.

3.1.1. Effect on LOR positioning. Figure 5 illustrates the effect of the asymmetrical component of a $y$-offset, which alters the position of the geometrical center, on the apparent 


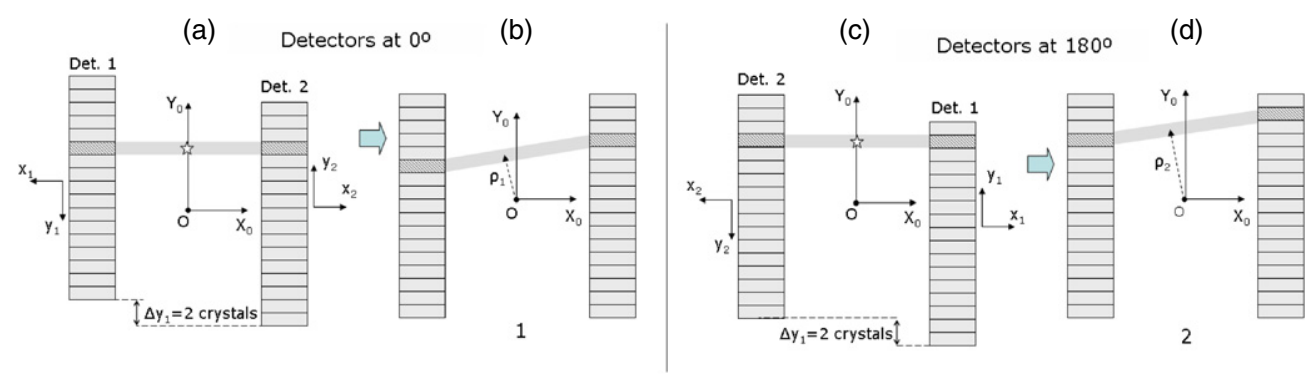

Figure 5. Drawing of a detector pair in two gantry positions that should theoretically generate coincident LORs. The star represents an annihilation event. Detector 1 has a large $y$-offset of $\Delta y_{1}=-3.2 \mathrm{~mm}$ (two-crystal widths) so that the effect is more conspicuous in the image. The panels labeled (a) and (c) show the LOR in the real position of the detectors, while the panels labeled (b) and (d) show the apparent LOR that would be obtained if the offset were ignored.

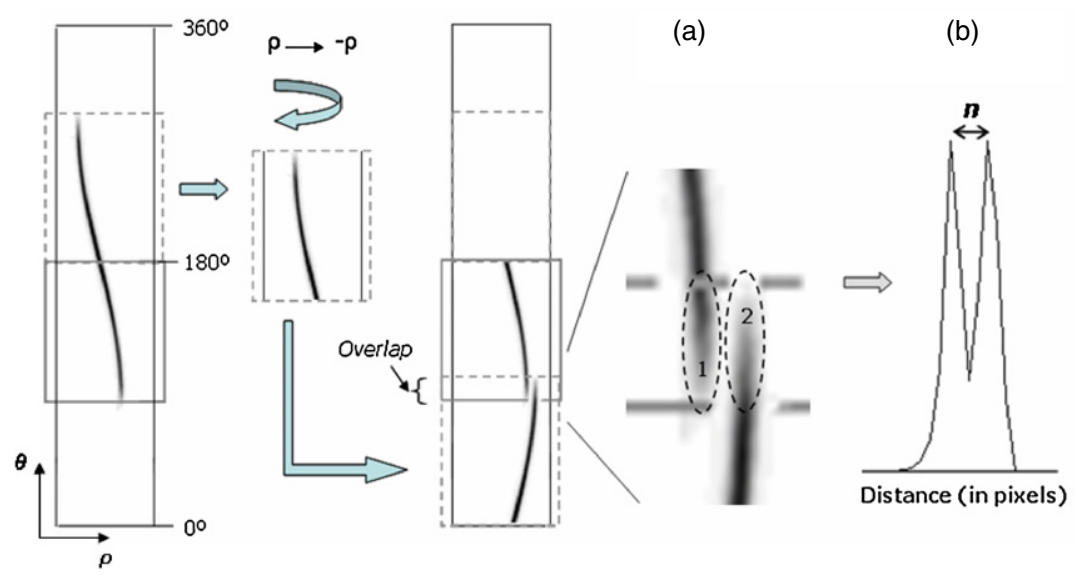

Figure 6. Example of a $y$-offset with an asymmetrical component (mismatch between the geometrical center and the COR). (a) Zoomed-in view of the overlap region, showing the radial gap. Labels 1 and 2 identify data acquired at gantry positions 1 (detectors at $0^{\circ}$ ) and 2 (detectors at $180^{\circ}$ ) in figure 5. (b) Radial profile across the gap region.

position of the LORs. The two gantry positions shown in the figure (corresponding to $0^{\circ}$ and $180^{\circ}$ of rotation) should theoretically generate equivalent LORs. However, if an asymmetrical $y$-offset is ignored, the counts in the indicated LORs will be assigned to erroneous bins in the corresponding 2D sinogram.

3.1.2. Effect on the $2 D$ sinogram. Figure 6 shows the consequence in our experimental setup of the type of LOR positioning error on the $180^{\circ} 2 \mathrm{D}$ sinogram mentioned above: the region of overlap (zoomed-in view in figure 6(a)) shows a discontinuity that we refer to as the 'radial gap' in the bins corresponding to gantry positions with $180^{\circ}$ of difference. This gap is only visible in the $14^{\circ}$ sector, which is scanned twice in one rotation, as explained above. Data on the left side of the radial gap ( 1 in figure 6(a)) correspond to events read while the detectors were at the $0^{\circ}$ gantry position, and data to the right of the radial gap ( 2 in figure $6(a)$ ) were 


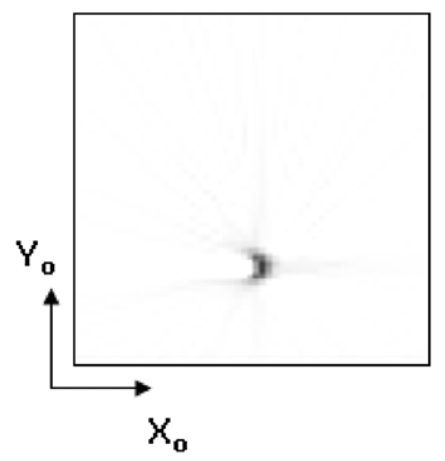

Figure 7. Effect of a $y$-offset on the reconstructed image: transaxial view of a simulated point source for a $y$-offset of $1.6 \mathrm{~mm}$ showing a 'crescent' shape.

acquired with the detectors at $180^{\circ}$. The $y$-offset, $\Delta y$, is directly related to the number of radial bins, $n$, in the sinogram spanned by the discontinuity, according to

$$
\Delta y=n \cdot b,
$$

where $b$ is the radial bin size in $\mathrm{mm}$ and $\Delta y$ is the $y$-offset. Due to the binning in the sinogram, $n$ is an integer value, and it can be seen from equation (2) that the estimate for $\Delta y$ will be discretized (size of the radial bin, $b$ ). The value $n$ only depends on the amount of mismatch between the geometric center and the COR, which is a function of the relative displacement between detectors. The 'sign' of the radial gap is related to the direction of the mismatch between the geometric center and the COR.

3.1.3. Effect on the reconstructed image. Figure 7 shows the effect of a mismatch between the COR and the geometric center on the reconstructed image of a point source, which is a 'crescent' shape of the image that deteriorates resolution. The simulation corresponds to a point source located at $10 \mathrm{~mm}$ from the center of the FOV in the central slice. The effect is the same irrespective of whether only one or both detectors are actually misaligned; for these reason, we will refer to these two situations that produce the same effect as 'equivalent'.

A summary of the effects and tolerance for the $y$-offset is presented in table 1 (rows 1 and 2).

\subsection{Misalignment along the $x$-axis with mismatch between geometric center and COR}

The misalignment along the $x$-axis, hereafter referred to as the ' $x$-offset', may also lead to two types of effect. The first one derives from a differential misalignment between opposite detectors and leads to a mismatch between the geometrical center and the COR ('asymmetrical component'). The second one occurs when both detectors are misplaced the same amount in opposite directions and leads to an erroneous value of the distance between detectors (see section 3.3).

This section describes the asymmetrical component. An $x$-offset that produces a change in the geometrical center induces LOR mispositioning in both the $x$ and $z$ coordinates (axial and transaxial views), which will be studied separately below. 
Table 1. Summary of the study of misalignments. For each misalignment type, the table indicates the simulation study used to assess its effect on the sinogram and on the reconstructed image, the tolerance formula (minimum value to produce a noticeable effect) and its value for the particular case of the rPET system.

\begin{tabular}{|c|c|c|c|c|c|}
\hline Misalignment & Acquisition & $\begin{array}{l}\text { Effect on } \\
\text { sinogram }\end{array}$ & $\begin{array}{l}\text { Effect on } \\
\text { image }\end{array}$ & Tolerance & $\begin{array}{l}\text { Value for the } \\
\text { rPET system }\end{array}$ \\
\hline $\begin{array}{l}y \text {-offset, } \\
\text { affecting COR } \\
\text { (asymmetrical) }\end{array}$ & $\begin{array}{l}\text { Non- } \\
\text { centered } \\
\text { point } \\
\text { source } \\
\end{array}$ & Radial gap & $\begin{array}{l}\text { Crescent } \\
\text { shape }\end{array}$ & Half pitch size & $0.8 \mathrm{~mm}$ \\
\hline $\begin{array}{l}y \text {-offset, not } \\
\text { affecting COR } \\
\text { (symmetrical) }\end{array}$ & $\begin{array}{l}\text { Non- } \\
\text { centered } \\
\text { point } \\
\text { source } \\
\end{array}$ & $\begin{array}{l}\text { Higher } \\
\text { FWHM in } \\
\text { coronal } \\
\text { view }\end{array}$ & $\begin{array}{l}\text { Transaxial } \\
\text { resolution } \\
\text { loss }\end{array}$ & Not considered & $\begin{array}{l}\text { Not } \\
\text { considered }\end{array}$ \\
\hline $\begin{array}{l}x \text {-offset, } \\
\text { affecting COR } \\
\text { (asymmetrical) }\end{array}$ & $\begin{array}{l}\text { Centered- } \\
\text { FOV point } \\
\text { source }\end{array}$ & $\begin{array}{l}\text { Axial gap } \\
\text { Center of } \\
\text { mass } \\
\text { modified } \\
\text { Higher } \\
\text { FWHM in } \\
\text { coronal } \\
\text { view }\end{array}$ & $\begin{array}{l}\text { Crescent } \\
\text { shape }\end{array}$ & $\Delta x=\left[\frac{(R+l / 2)}{(N / 2-1.5)}\right]$ & $6.9 \mathrm{~mm}$ \\
\hline $\begin{array}{l}x \text {-offset, not } \\
\text { affecting COR } \\
\text { (symmetrical) }\end{array}$ & $\begin{array}{l}\text { Non- } \\
\text { centered } \\
\text { point } \\
\text { source }\end{array}$ & $\begin{array}{l}\text { Higher } \\
\text { FWHM in } \\
\text { coronal } \\
\text { view }\end{array}$ & $\begin{array}{l}\text { Axial and } \\
\text { transaxial } \\
\text { resolution } \\
\text { loss }\end{array}$ & Not considered & $\begin{array}{l}\text { Not } \\
\text { considered }\end{array}$ \\
\hline z-offset & $\begin{array}{l}\text { Any point } \\
\text { source }\end{array}$ & $\begin{array}{l}\text { Higher } \\
\text { FWHM in } \\
\text { sagittal } \\
\text { view }\end{array}$ & $\begin{array}{l}\text { Axial } \\
\text { resolution } \\
\text { loss }\end{array}$ & Pitch size & $1.6 \mathrm{~mm}$ \\
\hline $\begin{array}{l}\text { Tilt (along } \\
y \text {-axis) }\end{array}$ & $\begin{array}{l}\text { Non } \\
\text { centered } \\
\text { point } \\
\text { source } \\
\end{array}$ & Radial gap & $\begin{array}{l}\text { Axial } \\
\text { resolution } \\
\text { loss }\end{array}$ & $\theta_{\min }=\arctan \frac{\text { pitch }}{(l / 2)}$ & $14.9^{\circ}$ \\
\hline $\begin{array}{l}\text { Slant (along } \\
z \text {-axis) }\end{array}$ & $\begin{array}{l}\text { Any } \\
\text { known } \\
\text { point } \\
\text { source } \\
\text { location } \\
\end{array}$ & $\begin{array}{l}\text { Higher } \\
\text { FWHM in } \\
\text { sagittal } \\
\text { view }\end{array}$ & $\begin{array}{l}\text { Crescent } \\
\text { shape }\end{array}$ & $\theta_{\min }=\arctan \frac{\text { pitch }}{(l / 2)}$ & $14.9^{\circ}$ \\
\hline Skew & $\begin{array}{l}\text { Non- } \\
\text { centered } \\
\text { point } \\
\text { source } \\
\text { (both in } \\
x-y \text { and } z \text { ) }\end{array}$ & $\begin{array}{l}\text { Radial gap } \\
\text { and higher } \\
\text { FWHM in } \\
\text { axial view }\end{array}$ & $\begin{array}{l}\text { Transaxial } \\
\text { and axial } \\
\text { resolution } \\
\text { loss }\end{array}$ & $\begin{array}{l}\xi_{\min }= \\
\arcsin \left(\frac{\text { pitch }}{R-\text { pitch } / 2}\right)\end{array}$ & $4.2^{\circ}$ \\
\hline
\end{tabular}

3.2.1. Effect on LOR positioning in the z coordinate (axial view). Figure 8 shows an example of an $x$-offset, $\Delta x$, in detector 2 . Gantry positions at $0^{\circ}$ and $180^{\circ}$ would show equivalent LORs if there were no offset $(\Delta x=0)$.

3.2.2. Effect on the $z$ coordinate of the sinogram (axial view). Figure 9 shows a plot of a $3 \mathrm{D}$ sinogram for coordinates $\theta$ (120 bins) and $s\left(28^{2}\right.$ bins). Note that the most oblique 2D sinograms containing the point source (A in figure 9 ) lack half of the angular positions. This 
(a) Detectors at $0^{\circ}$

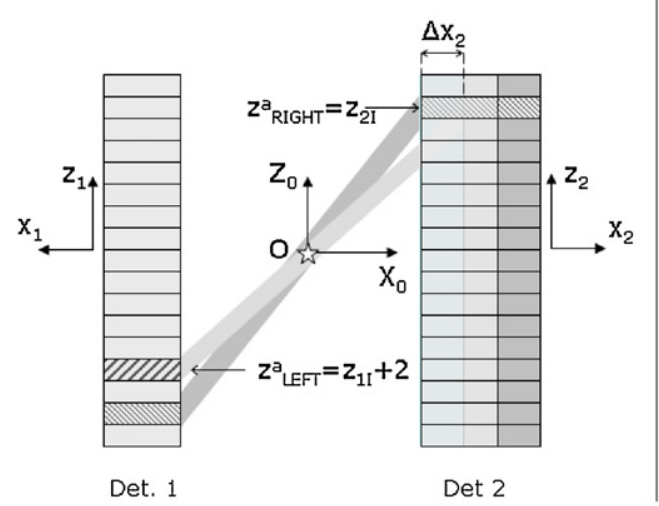

(b) Detectors at $180^{\circ}$

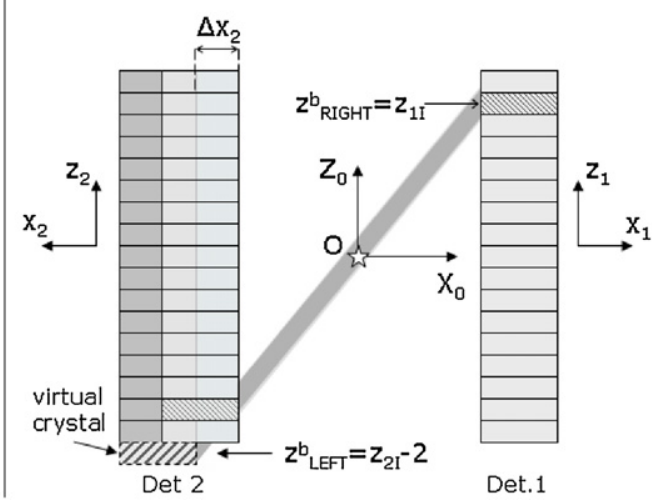

Figure 8. Axial view of a detector pair at $0^{\circ}$ (a) and $180^{\circ}$ (b) when there is an $x$-offset in detector 2. The ideal detectors (without misalignment) are depicted in light gray and the real detector (with misalignment) in dark gray. The star represents an annihilation event. The apparent LOR is depicted in light gray, while the real LOR is depicted in dark gray. For a given LOR, $z_{1 \mathrm{II}}$ and $z_{2 \mathrm{I}}$ are the apparent axial positions of the crystals that form the LOR in detectors 1 and 2 respectively. For a given axial position on the right detector, $z_{\text {RIGHT }}^{\mathrm{a}}$, the left detector crystals that define the equivalent LORs at gantry positions $0^{\circ}$ and $180^{\circ}, z^{\mathrm{b}} \mathrm{LEFT}$, have different axial positions.

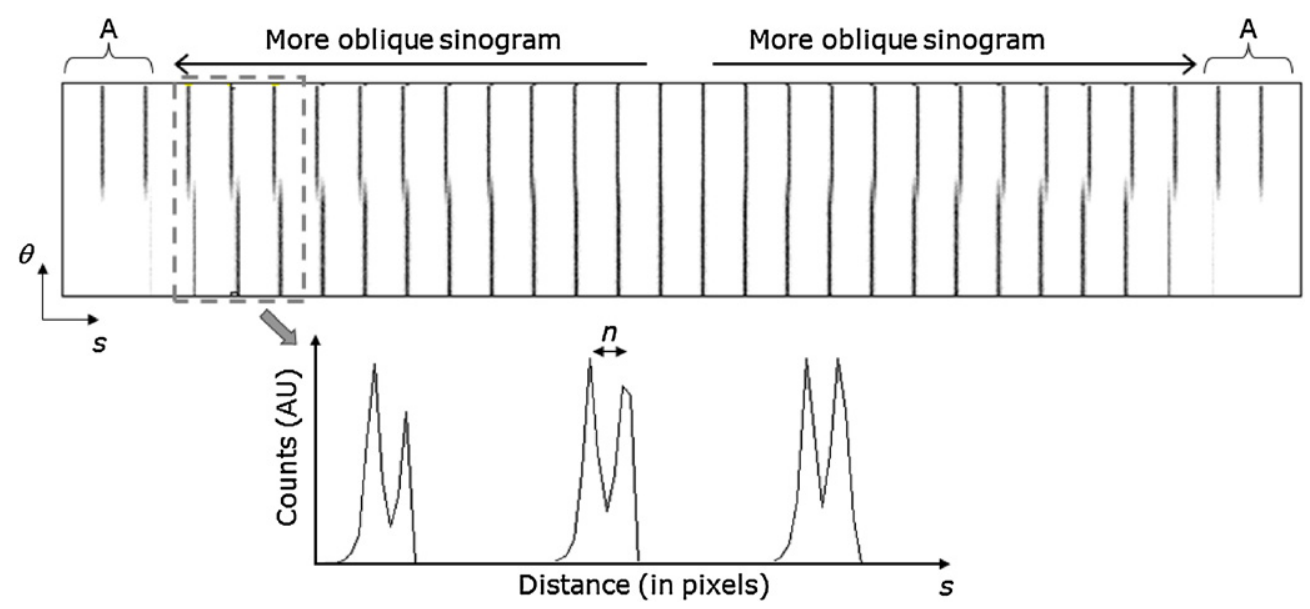

Figure 9. Top: plot of a 3D sinogram of a point source placed at the center of the FOV. Coordinates are $(\theta, s)$. Bottom: profiles along the $s$ axis corresponding to the highlighted area in the sinogram. Due to the $x$-offset, the gap increases for more oblique 2D sinograms.

is due to the fact that there is no opposite crystal to draw the LOR in those extreme positions (see figure 8 (b)).

Since the size of these discontinuities in the sinogram is directly related to the $x$-offset, it is possible to estimate this offset from the axial gaps measured on the sinograms. Figure 10 shows a zoomed-in picture of the top half of one detector, depicting the real and apparent detector positions. 


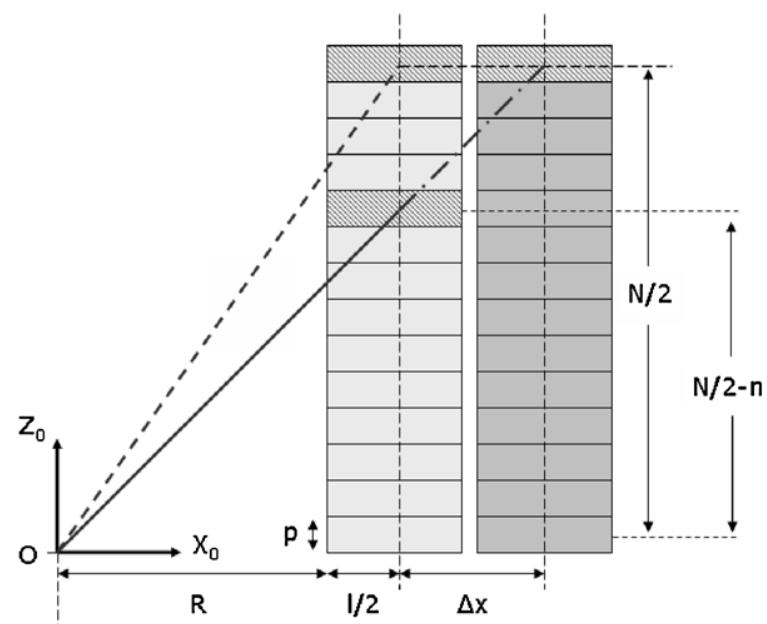

Figure 10. Diagram of the top half of one detector showing the rationale for the estimation of the $x$-offset. The detector in the ideal position is shown in light gray and the detector in the real position (with misalignment) is shown in dark gray. $R$ is the radius of rotation, $p$ the pitch size, $N$ the number of crystals in the axial direction, $l$ the crystal thickness and $\Delta x$ the $x$-offset of the detector.

The value of $\Delta x$ can be obtained from simple trigonometric relations. For the upper crystal row of the detector, we have

$$
\frac{p \cdot(N / 2-0.5)}{R+l / 2+\Delta x}=\frac{p \cdot(N / 2-0.5-n)}{R+l / 2},
$$

where $R$ is the radius of rotation, $N$ is the number of crystals in the detector along the $z$-axis, $l$ is the crystal thickness, $p$ is the pitch size and $n$ is the number of crystals by which the LOR is misplaced (equal to half the axial gap in sinogram bins). Thus, the value of $\Delta x$ can be calculated as

$$
\Delta x=(R+l / 2) \cdot\left[\frac{n}{(N / 2-0.5-n)}\right] .
$$

This effect will appear in the 3D sinogram if there is a minimum change of one crystal in the position of the LORs, i.e. $n=1$. Equation (4) shows that the minimum value for the calculated $x$-offset, $\Delta x$, to have an effect is about $7 \mathrm{~mm}$ for the rPET scanner.

Knowledge of the position of the detectors along the $x$-axis enables a software correction that will lead to correct positioning of the counts in the sinogram. Thus, good quality images can be reconstructed despite the physical mismatch between the geometrical center and the COR. The gap size can be used to obtain a rough estimate of the asymmetrical component of an $x$-offset, as explained above. In order to refine this estimate, we can use the effect on the 'transaxial view' (see the following section).

3.2.3. Effect on LOR positioning in the $x$ coordinate (transaxial view). In the case of an asymmetrical $x$-offset, the LORs corresponding to the initial and final rotating positions (gantry at $0^{\circ}$ and $180^{\circ}$ ), which should be equivalent, will show the same $\theta$ value but a different $\rho$ value in the 2D sinogram (figure 11(c)). 

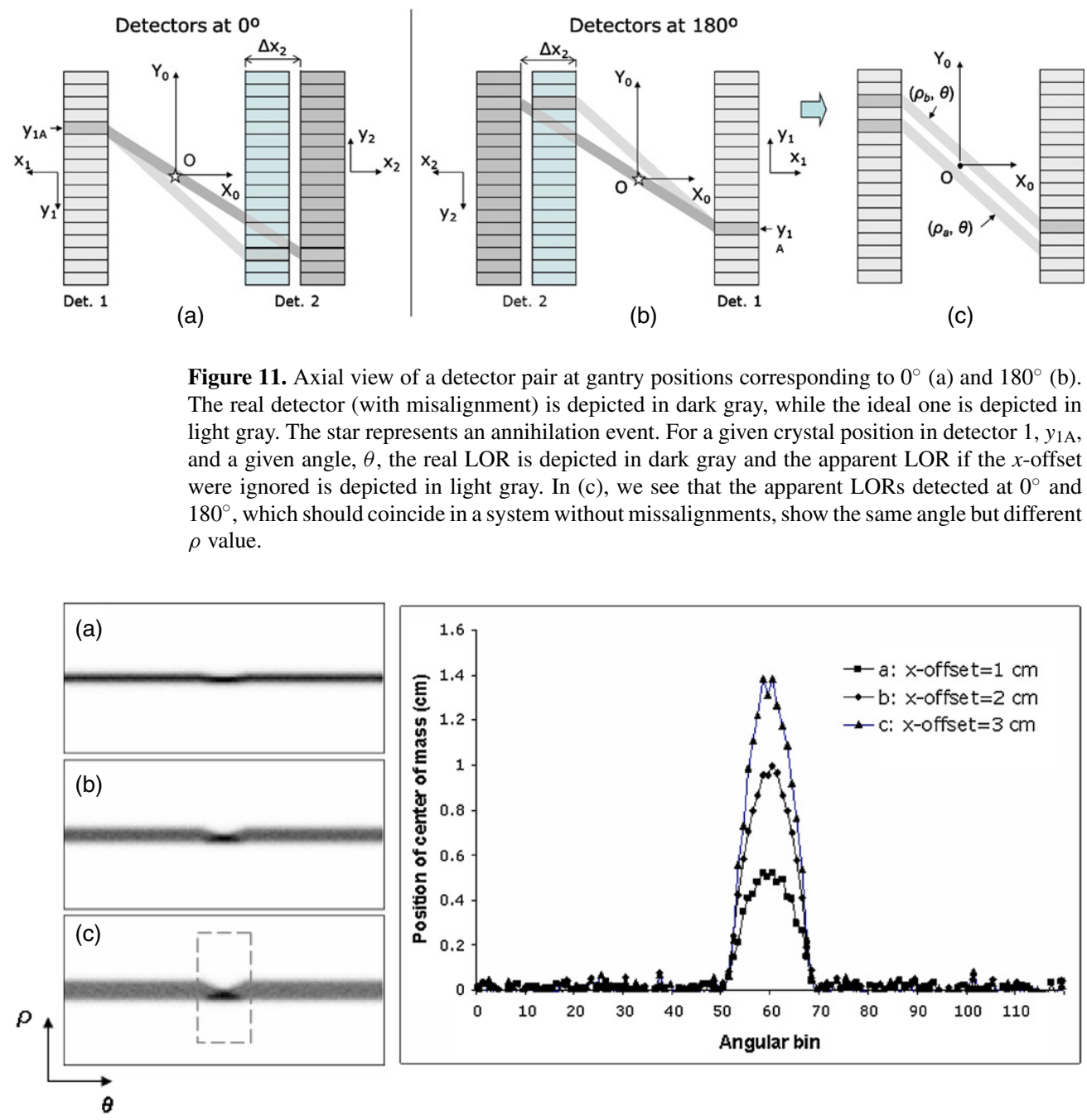

Figure 12. Left: sum of all oblique 2D sinograms for different $x$-offset values $\{1,2,3 \mathrm{~cm}\}$. Such large values were used so the effect is more conspicuous in the image. Right: center of mass vector for each $2 \mathrm{D}$ sinogram on the left.

3.2.4. Effect on the $3 D$ sinogram in the $x$ coordinate (axial view). The error in the LOR positioning explained above results in a widening of the $2 \mathrm{D}$ sinogram. In our experimental setup, due to the arrangement in a $180^{\circ}$ sinogram, the sinogram area corresponding to the initial and final gantry positions shows a characteristic shape (gray dotted square in figure 12, left). The right panel of figure 12 shows that the trajectory followed by the center of mass along the $\rho$-coordinate presents a deviation from the expected profile at the overlap area. The amount of deviation corresponds to half the differential $x$-offset of the system. This value also reflects the difference between the COR and center of the FOV.

3.2.5. Effect on the reconstructed image. The visual effect on the reconstructed image of a point source is similar to that produced by a $y$-offset (a 'crescent' shape). As mentioned 


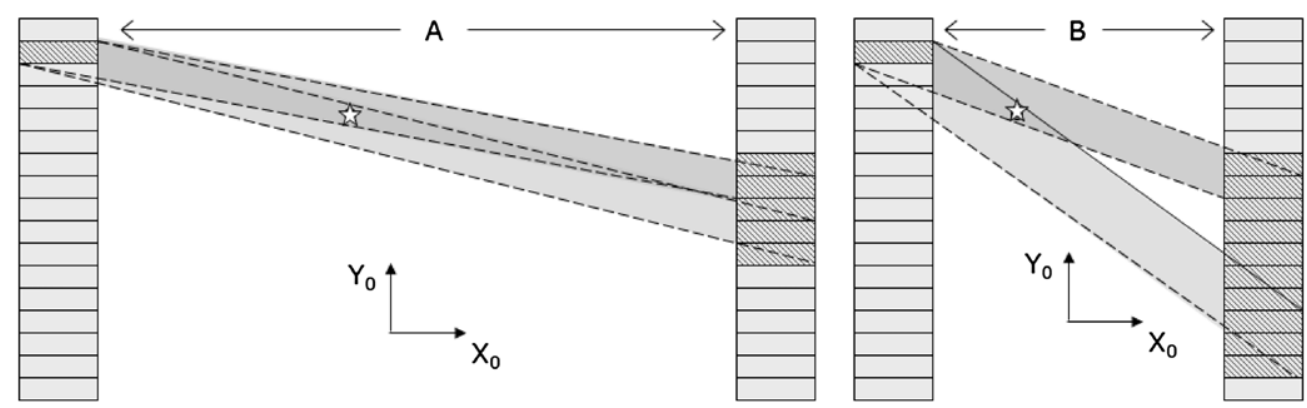

Figure 13. Transaxial view of one pair of detectors at different distances. Given an annihilation event (star), the dotted lines show the first and last tubes of response (TOR) corresponding to a single crystal (shadowed) in the left detector that would 'see' the point source. The dark gray TOR corresponds to the uppermost crystal and the light gray one to the lowest crystal. When the distance between detectors is $A$, the number of TORs is five (left panel), whereas for a distance of $B$ the number of TORs is ten (right panel).

before, the effect is the same for the equivalent situations, irrespective of whether only one or both detectors are actually misaligned.

\subsection{Effects of erroneous calibration of the distance between detectors}

To study the effect of erroneous calibration of the distance between detectors, we considered the particular situation which arises when both detectors are misplaced by the same distance in opposite directions on the $x$-axis, that is, with no change in the geometrical center (symmetrical offset). In this case, an erroneous value for the distance between detectors only leads to changes in the resolution of the reconstructed image.

This change in resolution is easily explained by considering that closer detectors subtend a larger solid angle for a point source. Figure 13 provides a transaxial view of a detector pair for two different values of the distance between detectors, showing that more tubes of response (TOR) intersect the point source when the detectors are closer. Therefore, if the actual distance between detectors is lower than the nominal one, the point source seen by the same number of crystals would be larger, thus showing a wider point spread function (PSF).

Figure 14 shows the effect of erroneous calibration on the resolution of the reconstructed image, for three different distances between detectors (symmetrical misalignment, no COR mismatch). The simulation corresponds to a point source located at the center of the FOV in the central slice. The PSF increases when the actual distance between detectors is underestimated (figures 14(b) and (c)).

A summary of the effects and tolerance for the $x$-offset is presented in table 1 (rows 3 and 4).

\subsection{Misalignment along the z-axis}

The effect of erroneous calibration of the position of the detectors along the $z$-axis (' $z$-offset') is an incorrect positioning of the LORs in the axial direction that assigns the counts to an erroneous oblique 2D sinogram. This effect can be observed by tracking the trajectory followed by the planar projection of a point source after rebinning the sinogram data with single-slice rebinning algorithm (SSRB) (span 2). Ideally, the point source should appear at 

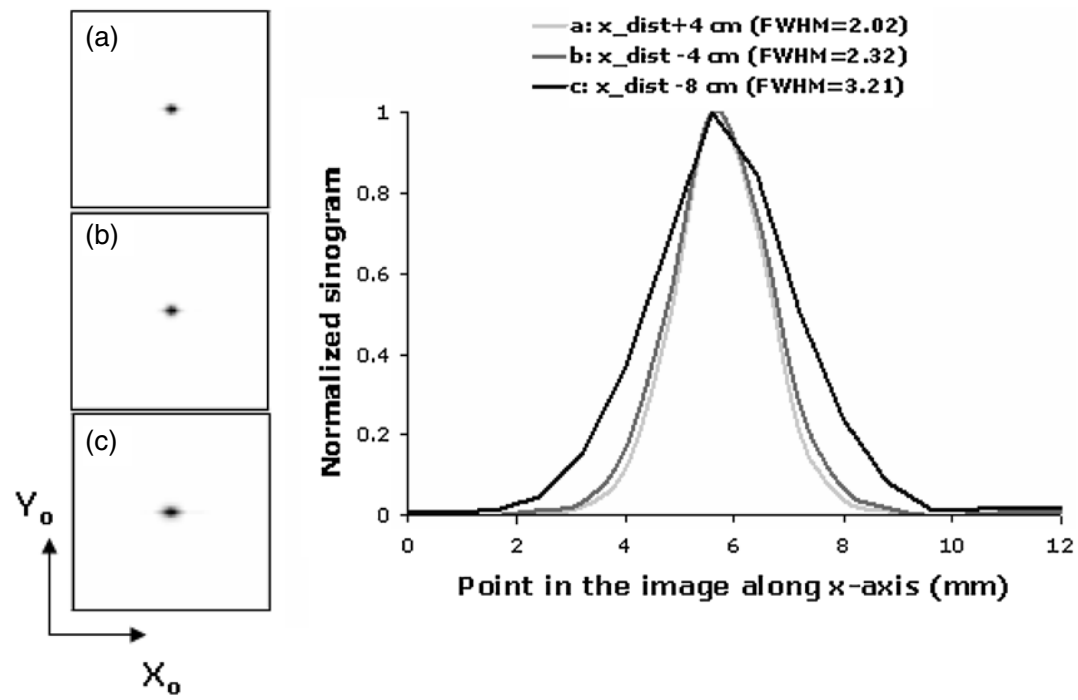

Figure 14. Effect of an erroneous calibration of the distance between detectors on the resolution of the reconstructed image. Left: coronal view of a simulated point source for errors in the distance between detectors of $4,-4$, and $-8 \mathrm{~cm}$. Right: profiles drawn in the reconstructed images along the $X_{0}$-axis.

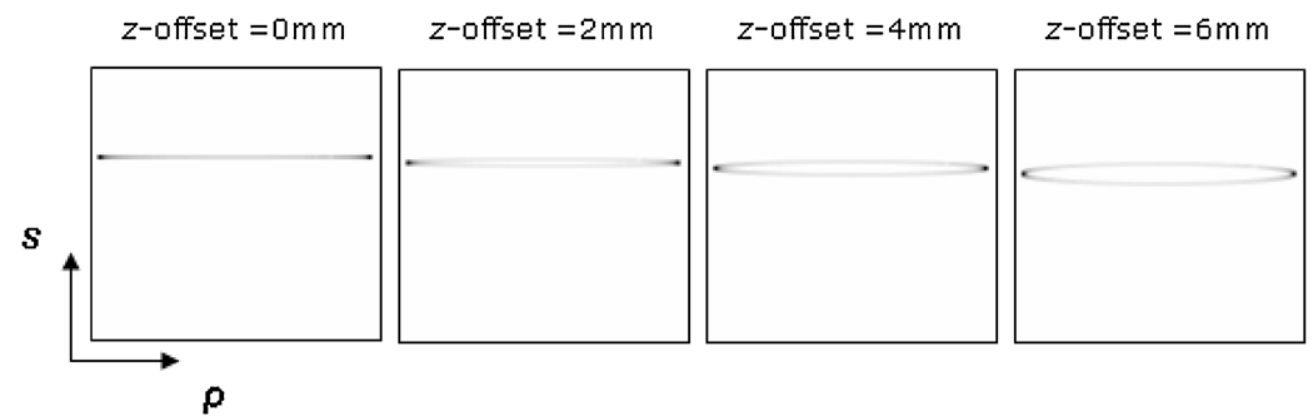

Figure 15. Sum of projection angles covering $360^{\circ}$ for four different $z$-offsets: note the ellipsoidal trajectory.

the same position along the $s$-axis. Thus, the result of adding up all the projections should be a horizontal straight line.

3.4.1. Effect on LOR positioning. To show the effect of this offset, we simulated a complete set of projections of a point source located away from the center of the FOV for a highresolution system with an intrinsic resolution of $0.4 \mathrm{~mm}$, for different values of $z$-offset (figure 15).

Figure 16 shows that an annihilation event close to the detector will generate projections at $0^{\circ}$ and $180^{\circ}$ with an axial difference equal to the $z$-offset. 
(a) Detectors at 00

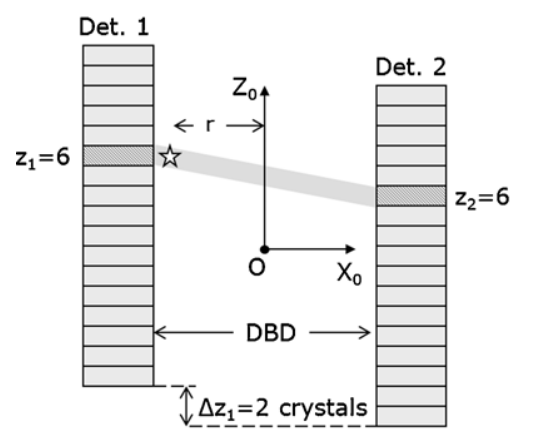

(b) Detectors at $180^{\circ}$

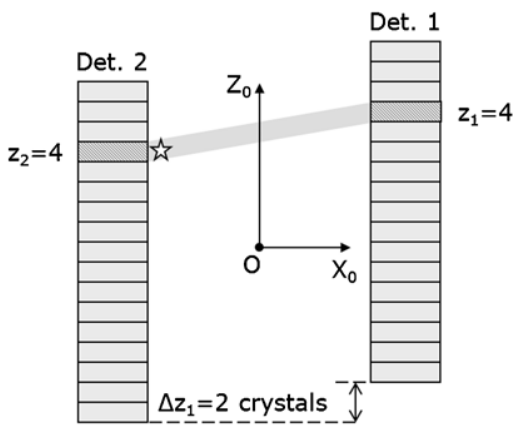

Figure 16. Axial view of a detector pair at $0^{\circ}$ (a) and $180^{\circ}$ (b) when there is a $z$-offset of $\Delta z_{1}$ in detector 1. The star represents an annihilation event. When the gantry is at the starting position (a), the 'direct' LOR that contains the event is different from the one at $180^{\circ}$. For this reason, projection at different angles will show the point source at different axial positions.

For annihilation events originated at a distance $r$ from the axis of rotation, we can derive a formula to estimate the $z$-offset, by applying simple trigonometric relations, as follows:

$$
\frac{z-\text { offset }}{\mathrm{DBD}}=\frac{\text { axialdiff }}{\left(\frac{\mathrm{DBD}}{2}+r\right)} \Rightarrow z-\text { offset }=\mathrm{DBD} \cdot \frac{\text { axialdiff }}{\left(\frac{D B D}{2}+r\right)},
$$

where DBD is the distance between detectors, $r$ is the distance to the COR, and axialdiff is the length of the short axis of the ellipsoidal trajectory.

Since the achievable resolution in the projections with the scanner under study is only about $0.8 \mathrm{~mm}$, the ellipsoidal shape of the trajectory is barely visible. Thus, the effect of a $z$-offset in practice will appear as an increase in the FWHM of the profile along the $z$-axis. This increase will only be visible when there is a change in the axial positions of the LORs that requires a $z$-offset higher than the crystal pitch.

A summary of the effects and tolerance for the $z$-offset is presented in table 1 (row 5).

\subsection{Angular misalignments}

As shown in the left panel of figure 3, the detector may have angular misalignments around the three axes, $X_{0}, Y_{0}, Z_{0}$.

3.5.1. Effect of slant and tilt. Both slant and tilt, $(\gamma$ and $\sigma$ in figure 3$)$, angular rotations around $Y_{\mathrm{o}}$ and around $Z_{\mathrm{o}}$ respectively, produce the same effect in a system with planar detectors. From figure 17, we can see that the minimum value of $\sigma$ that would generate a notable effect, i.e. that would change the identification of the crystals involved in the LOR, is given by

$$
\tan \left(\sigma_{\min }\right)=\frac{\text { pitch }}{l / 2}
$$

where pitch is the distance between the center of two adjacent crystals and $l$ is the crystal length.

For the scanner under study, with a crystal pitch of $1.6 \mathrm{~mm}$ and $l=12 \mathrm{~mm}$, we obtain $\theta_{\min }=14.9^{\circ}$. Such a large angular error is highly unlikely to be found in real equipment after manufacturing controls. 

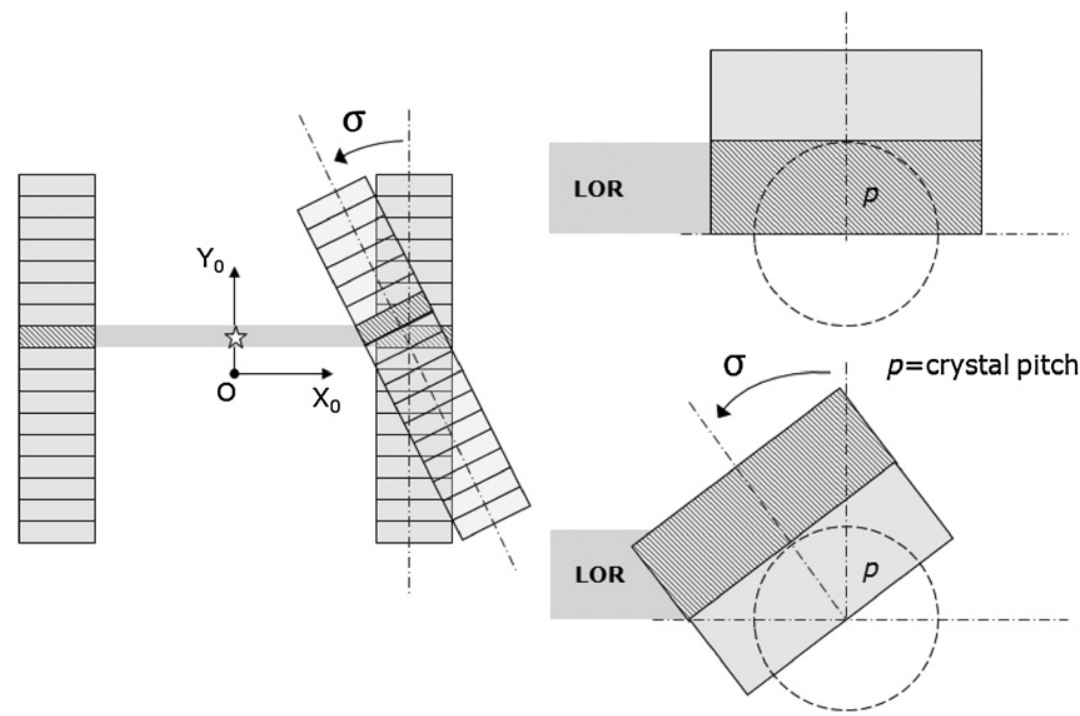

Figure 17. Minimum detector tilt required to produce a notable effect (change in LOR). Left: axial view of a detector pair with misalignment in $\sigma$. The star represents an annihilation event. Right: zoomed-in view of one detector showing the crystal that detects an annihilation event (shaded) in the ideal position (top) and the real position with misalignment (bottom).

3.5.2. Effect of skew. In the case of detector skew (rotation around the $x$-axis, $\xi$ in figure 3), an erroneous pixel assignment results in a loss in the $z$-resolution and a visual effect similar to that described for a mismatch of the COR and the geometric center ('crescent' shape).

As shown in figure 18, the effect becomes more severe for peripheral points in the FOV. For the detector pixel shadowed in figure 18, we can see that the angle that will cause a change in the crystal assignment of the LOR, given by equation (7), is about $4^{\circ}$ for the rPET system.

$$
\xi_{\min }=\arcsin \left(\frac{\text { pitch }}{N \cdot \text { pitch } / 2-\text { pitch } / 2}\right)=\arcsin \left(\frac{1.6}{21.6}\right)=4.2^{\circ},
$$

where $N$ is the number of crystals in the detector along the $z$-axis.

Due to the way the detector is attached to the gantry (figure 3, left), the only angulation that may be relevant in practice is the one around the $z$-axis, which is guaranteed to be much smaller than the calculated $\theta_{\min }\left(14.9^{\circ}\right)$ after the mechanical calibration. Misalignment in the other angles would only be due to the looseness of the crystal matrix inside the detector box, an effect that can be considered negligible (definitely below the minimum values calculated previously). For this reason, angular misalignments of the detector are not taken into account in the calibration algorithm. A summary of the effects and proposed method tolerance for the angular misalignments is presented in table 1 (rows 6, 7 and 8).

\subsection{Summary}

Table 1 summarizes our results, indicating the formula for the minimum value necessary to produce a visible effect (hereafter 'tolerance') and the value for the particular case of the rPET system studied. 


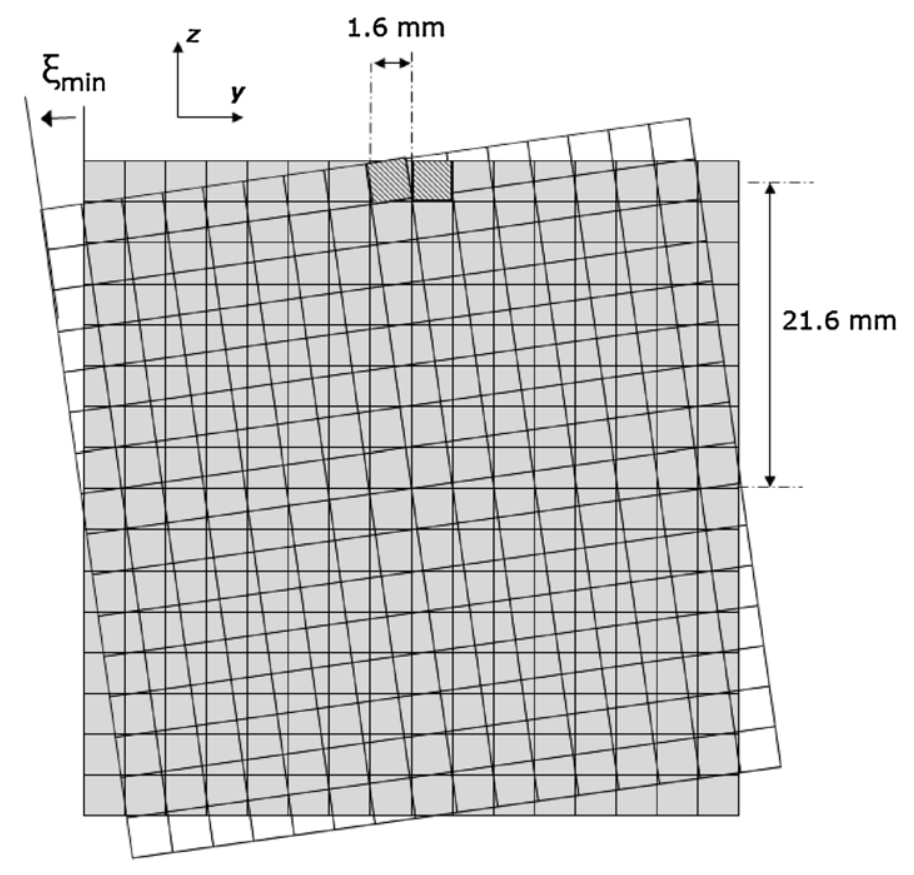

Figure 18. Rotation of one detector around the $x$-coordinate.

All the misalignments in table 1 correspond to a single pair of detectors. In a system with more than one pair of detectors, such as the one under study here, there are two additional calibration parameters to measure: the $z$-offset between detector pairs and the angle between detector pairs (angle $\Phi_{\text {offset }}$ in figure 3, right). We did not perform any simulation of these two misalignments, since the procedure to obtain their values is straightforward (explained below).

\section{Calibration algorithm}

The results of this study enabled us to develop a calibration algorithm to calculate scanner misalignments. The input data for the algorithm are two acquisitions in coincidence list mode of a small spherical ${ }^{22} \mathrm{Na}$ point source (in our case, $0.5 \mathrm{~mm}$ in diameter, encapsulated in a $1 \mathrm{~cm}^{3}$ epoxy box). In the first acquisition, the source is placed at the center of the FOV ('centered acquisition') and in the second one the source is located away from the center in the transaxial plane ('non-centered acquisition'). Reconstructions are generated by means of SSRB with span 1, followed by a 2D filtered back-projection (FBP) with a ramp filter. For the rPET scanner, the voxel size of the resulting image is $0.8 \mathrm{~mm}$ isotropic. The result of the calibration algorithm is a file containing the misalignment values. This file is used by subsequent reconstructions to generate a corrected sinogram, in which the position of each LOR is relocated according to the actual misalignments.

For each offset, the program makes a first estimate and guides the user through an interactive tuning procedure. After an initial estimation of all the offsets, in a process called coarse tuning, the software offers the user the possibility of performing more iterations of the calibration algorithm using smaller offset values (fine tuning) until the result is satisfactory. 

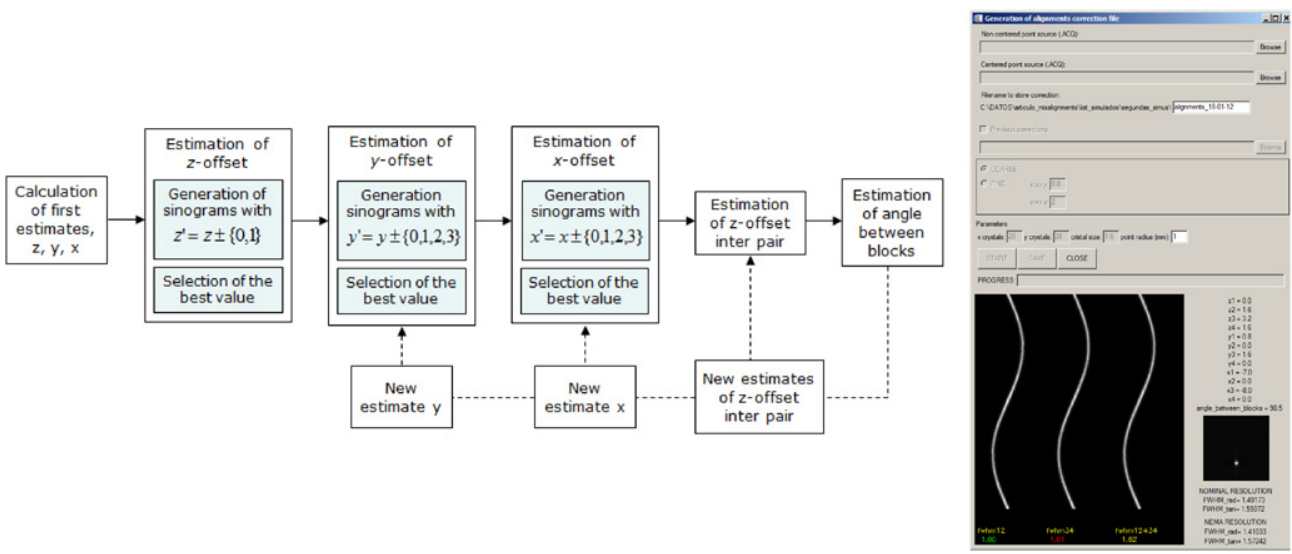

Figure 19. Left: flowchart of the calibration algorithm, where $x^{\prime}, y^{\prime}$ and $z^{\prime}$ are the detector coordinates after correction. Dotted lines indicate iterative paths for a fine tuning of the offset values. Right: interface of the calibration tool at the end of the process showing the sinogram from each pair of detector and the summed sinogram, the misalignment values found, and the resulting resolution.

When this process has finished for all the possible offsets, the final values are stored. These offset values are used at the reconstruction phase to define the correct positions of the LORs: the corrected $x^{\prime}, y^{\prime}$ and $z^{\prime}$ coordinates will be the nominal values plus the calculated offset.

Figure 19 shows a flowchart of the calibration process and the interface of the calibration tool at the end of the process.

The following subsections present a detailed explanation of the processing performed at each step of the flow chart.

\subsection{Estimation of the z-offset}

For the measurement of the $z$-offset between the two detectors of a pair, the calibration protocol makes use of the centered acquisition and generates sinograms for three different values of the detector 1 position along the $z$-axis, $z_{1}^{\prime}$ as follows:

$$
z_{1}^{\prime}=z_{1}+a \cdot z_{-} \text {offset }_{1} \quad a=\{0, \pm 1\},
$$

where $z_{1}$ is the nominal axial position of detector 1 and $z_{-}$offset $_{1}$ is the tentative value for $z$-offset selected by the user (the default value is the pitch size). The projection data are rebinned into a set of direct sinograms, with coordinates $(\rho, \theta, s)$, using SSRB with span 1, and the planar projections for each angle are added together to form an image of $55 \times 55$ pixels ${ }^{2}$. The optimum $z_{-}$offset $_{1}$ is obtained as the one that yields the lowest FWHM along both the $s$-axis and the $\theta$-axis of the rebinned sinogram (figure 20).

\subsection{Estimation of the $y$-offset}

The $y$-offset is estimated using data from the non-centered acquisition. The algorithm generates a first sinogram considering that all offsets but the $z$-offset, calculated in the previous step, is equal to zero and measures the sinogram radial gap using a procedure explained below for the gap size parameter. As we mentioned above, the observed sinogram gap is the same irrespective of whether only one detector is misaligned or both. For this reason, we explore sinograms considering the misaligned detector is the detector 1. 


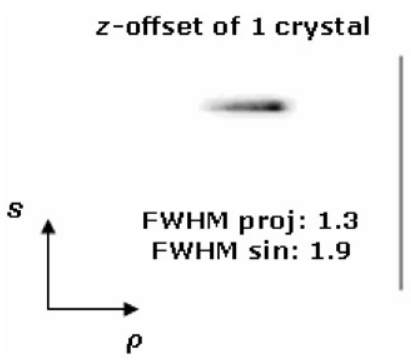

Figure 20. Images obtained for different $z$-offset values in the first detector when adding together the planar projections for every angle of one detector pair.

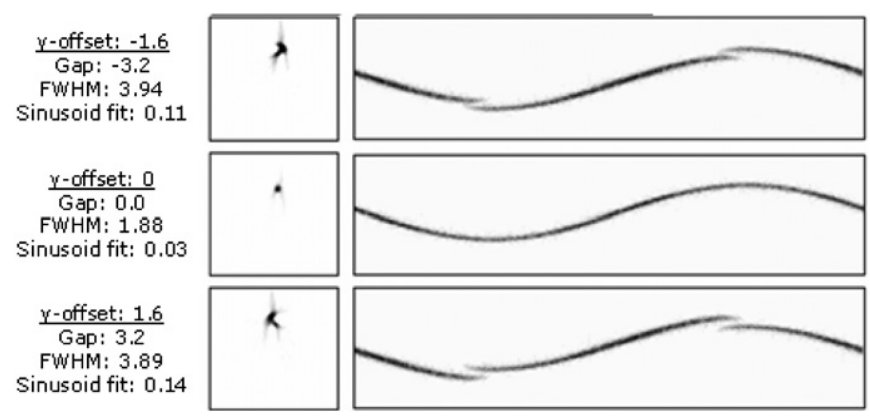

Figure 21. Estimation of the $y$-offset. Example of calculated values for 'gap size', 'sinogram FWHM' and 'sinusoid fitting score' (left), reconstructed image (middle) and sinograms (right) for different values of $y$-offset.

A first estimation of the $y$-offset is then calculated from this value according to equation (2), and the nominal value is corrected (obtaining $\hat{y}_{1}$ ). At this point, the algorithm generates sinograms corresponding to seven different positions of detector 1 along the $y$-axis, $y_{1}^{\prime}$ with

$$
y_{1}^{\prime}=\hat{y}_{1} \pm a \cdot \text { step }, \quad a=\{0,1,2,3\},
$$

where the step is chosen by the user (default value is a quarter of the pitch). We define three quality parameters (figures of merit) to guide the selection of the $y$-offset: the sinogram FWHM, the sinusoid fitting score and the gap size. To obtain the sinogram FWHM, the algorithm adds up all the rows in the sinogram (each row represents data at one angle bin, $\theta$ ) with their center of mass aligned and fits the resulting profile to a Gaussian function. The sinusoid fitting score is computed as the root mean square error between the normalized profile given by the trajectory of the center of mass at each $\theta$ and an ideal sinusoid. Finally, the algorithm computes the position of the maximum for each angular bin and calculates the distance between the positions of the maximum value for every two consecutive angular bins, obtaining the gap size as the mean value of all the distances that are higher than one radial bin (this will correspond to the overlap area in figure 6(a)). The most appropriate $y$-offset is selected by the user based on the simultaneous visual inspection of the sinogram and the reconstructed image, as well as on the total of the three values of the figures of merit (figure 21). 


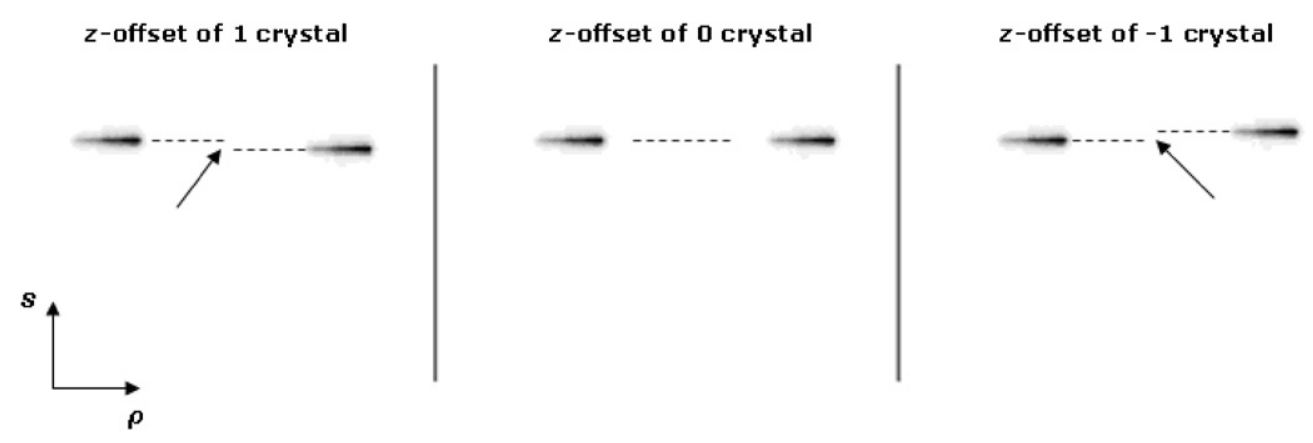

Figure 22. Images obtained by adding up the planar projection for each angle of the two detector pairs, for different values of the $z$-offset between detectors.

\subsection{Estimation of the $x$-offset}

The calibration protocol estimates the $x$-offset using the centered acquisition data. The algorithm generates a sinogram corrected for the offsets already estimated ( $z$ - and $y$-offsets) and provides an initial estimation of the $x$-offset according to equation (4) and the axial gap size in the sinogram.

The algorithm then generates sinograms corresponding to seven different positions of detector 1 along the $x$-axis, $x_{1}^{\prime}$, assuming that the position of detector 2 along the $x$-axis is correct (thus, ignoring the symmetrical component), as follows:

$$
x_{1}^{\prime}=\hat{x}_{1} \pm a \cdot \text { step, } \quad a=\{0,1,2,3\},
$$

where $\hat{x}_{1}$ is the ideal value corrected with the initial estimate of the $x$-offset and the step is chosen by the user (default value is $2 \mathrm{~mm}$ ).

For each sinogram, the algorithm calculates two figures of merit: the sinogram FWHM, explained above, and the deviation of the center of mass shown in figure 12, right. The selection of the appropriate $x$-offset is made by the user based on the visual inspection of the sinogram, the reconstructed image and the figures of merit defined, for each tested value.

\subsection{Estimation of the z-offset between detector pairs}

Once each pair of detectors is corrected for the $x$ - and $y$-offsets, the calibration algorithm determines the $z$-offset between detector pairs using data from the non-centered acquisition. For each detector pair, the algorithm generates an image by adding up the planar projections for each angle. The $z$-offset between detector pairs is calculated as the difference between the center of mass along the $z$-axis for each image, as shown in figure 22 .

\subsection{Estimation of the angle between detector pairs}

The last step is to determine the angle between detector pairs, which theoretically is $90^{\circ}$ (angle $\Phi_{\text {offset }}$ in figure 3, right). Using data from the non-centered acquisition, the algorithm compares the trajectory drawn by the center of mass for each angle bin (along the $\theta$ coordinate) of one detector pair with that obtained from other detector pair with different shift values, $\theta_{\text {shift }}$. The shift value that leads to the least error (first column in figure 23), two coincident trajectories 


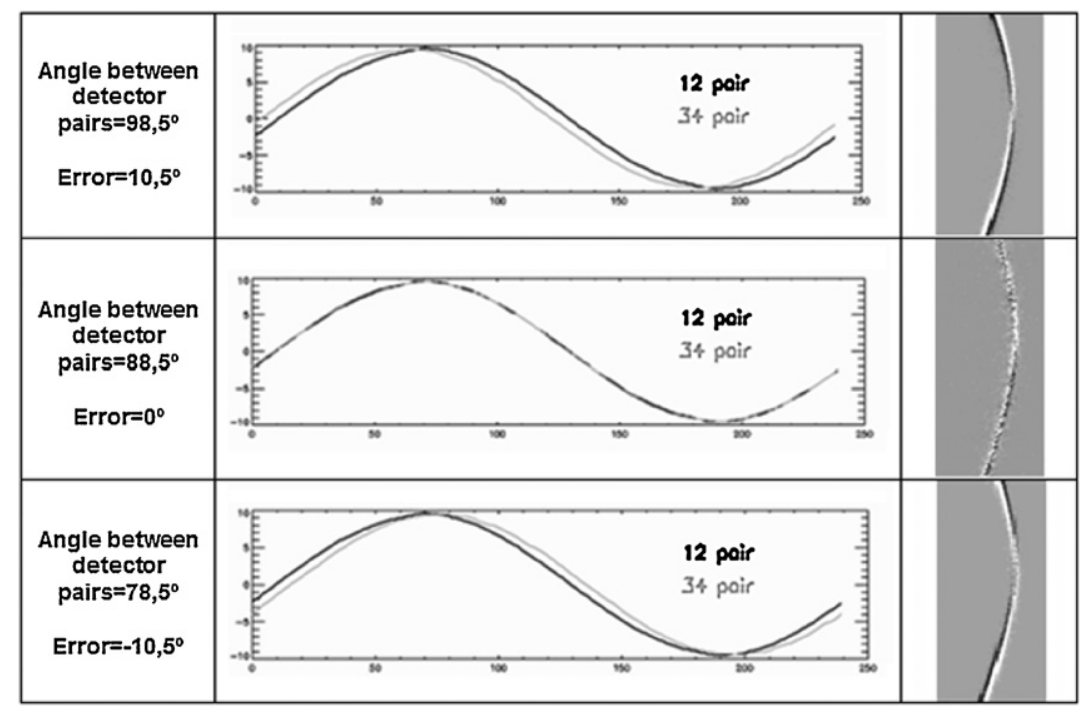

Figure 23. Center of mass trajectory for each detector pair and difference image of both sinograms. The central row shows the optimum value and the top and bottom rows show large offset values to make the effect more conspicuous in the image.

(second column in figure 23) and two coincident sinograms (third column in figure 23) is selected as the $\Phi_{\text {offset }}$.

\section{Evaluation}

As mentioned above, the method proposed only detects absolute misalignments, irrespective of whether the misalignments were produced by one or both detectors within the pair. We used the Hot Derenzo Phantom simulation described in section 2 to estimate the effect of the uncertainty provided by the method in these equivalent situations. The misalignments used are listed below ( $z$-offsets are set to zero given that they do not play a role in this problem):

$$
\begin{aligned}
& \Delta x_{1}=0.0, \Delta x_{2}=5.0, \Delta x_{3}=2.5, \Delta x_{4}=-2.5, \\
& \Delta y_{1}=0.0, \Delta y_{2}=1.6, \Delta y_{3}=0.8, \Delta y_{4}=-0.8, \\
& \Delta z_{1}=0.0, \Delta z_{2}=0.0, \Delta z_{3}=0.0, \Delta z_{4}=0.0, \\
& \Phi_{\text {offset }}=88.0 .
\end{aligned}
$$

An additional simulation of the phantom was performed without any misalignment in order to check the accuracy of the different corrections studied. The correction cases are described in table 2. All the misalignments were properly corrected in each case except those listed in column 3, which correspond to equivalent corrections. Option 1 is the equivalent correction when assuming that both detectors are misaligned and option 2 is the correction assuming only one detector is misaligned.

For each case, we computed the root mean square error (RMSE) inside the FOV relative to the maximum value of the image without misalignments and the peak-to-valley ratio measured in profiles along a row of rods of $1.6 \mathrm{~mm}$ (see profiles in figure 24). The analysis was performed by adding up 45 slices of the reconstructed image.

We can see that the relative RMSE decreases when the exact correction of the misalignments is applied, with no significant changes between the different equivalent 
Table 2. Results on reconstructed images from the Hot Derenzo Phantom simulation. For each case, the table indicates a brief description of the analyzed image, the estimated misalignment values used in the reconstruction, the computed RMSE relative to the maximum value of the image without misalignments and the peak-to-valley ratio measured in profiles along a row of rods of $1.6 \mathrm{~mm}$.

\begin{tabular}{|c|c|c|c|c|}
\hline Study name & Description & $\begin{array}{l}\text { Estimated } \\
\text { misalignments } \\
(\mathrm{mm})\end{array}$ & $\begin{array}{l}\text { Relative RMSE } \\
(\%)\end{array}$ & $\begin{array}{l}\text { Peak-to-valley } \\
\text { ratio }(1.6 \mathrm{~mm} \\
\text { rods) }\end{array}$ \\
\hline $\begin{array}{l}\text { Without } \\
\text { misalignments }\end{array}$ & $\begin{array}{l}\text { No } \\
\text { misalignments } \\
\text { were simulated }\end{array}$ & - & 0.0 & 2.9 \\
\hline $\begin{array}{l}\text { Without any } \\
\text { correction }\end{array}$ & $\begin{array}{l}\text { None of the } \\
\text { misalignments } \\
\text { were corrected }\end{array}$ & No corrections & 11.2 & 2.1 \\
\hline $\begin{array}{l}\text { No } x-y \text { correction } \\
\text { (pair } 1 \text { ) }\end{array}$ & $\begin{array}{l}\text { Misalignments } \\
\text { in detector pair } 1 \\
\text { (detectors } 1 \text { and } \\
\text { 2) were not } \\
\text { corrected }\end{array}$ & $\begin{array}{l}\Delta x_{1}=0.0 \\
\Delta x_{2}=0.0 \\
\Delta y_{1}=0.0 \\
\Delta y_{2}=0.0\end{array}$ & 7 & 1.8 \\
\hline $\begin{array}{l}\text { Misalignments } \\
\text { corrected }\end{array}$ & $\begin{array}{l}\text { All the } \\
\text { misalignments } \\
\text { were corrected }\end{array}$ & - & 2.4 & 2.7 \\
\hline$x$ corrected $-y($ opt 1$)$ & $\begin{array}{l}\text { Equivalent } \\
\text { correction } \\
\text { (option 1) for } y\end{array}$ & $\begin{array}{l}\Delta y_{1}=-0.8 \\
\Delta y_{2}=0.8\end{array}$ & 2.6 & 2.7 \\
\hline$x$ corrected $-y($ opt 2$)$ & $\begin{array}{l}\text { Equivalent } \\
\text { correction } \\
\text { (option } 2 \text { ) for } y\end{array}$ & $\begin{array}{l}\Delta y_{1}=0.0 \\
\Delta y_{2}=-1.6\end{array}$ & 2.9 & 2.7 \\
\hline$y$ corrected $-x($ opt 1$)$ & $\begin{array}{l}\text { Equivalent } \\
\text { correction } \\
\text { (option 1) for } x\end{array}$ & $\begin{array}{l}\Delta x_{1}=-2.5 \\
\Delta x_{2}=2.5\end{array}$ & 2.4 & 2.8 \\
\hline$y$ corrected $-x($ opt 2$)$ & $\begin{array}{l}\text { Equivalent } \\
\text { correction } \\
\text { (option 2) for } x\end{array}$ & $\begin{array}{l}\Delta x_{1}=-5.0 \\
\Delta x_{2}=0.0\end{array}$ & 2.4 & 2.8 \\
\hline$x($ opt 1$)-y($ opt 1$)$ & $\begin{array}{l}\text { Equivalent } \\
\text { correction } \\
\text { (option 1) for } \\
x \text { and } y\end{array}$ & $\begin{array}{l}\Delta x_{1}=-2.5 \\
\Delta x_{2}=2.5 \\
\Delta y_{1}=-0.8 \\
\Delta y_{2}=0.8\end{array}$ & 2.7 & 2.7 \\
\hline
\end{tabular}

corrections in the $x$ and $y$ coordinates (or both of them) and the exact one. These results show that an equivalent correction in the $x$ and $y$ coordinates does not affect the final quality of the image. Nevertheless, we can see important differences in the case where the misalignments were not corrected.

The same effect is observed when studying image profiles along a row of rods of $1.6 \mathrm{~mm}$ on the Hot Derenzo Phantom images, plotted in figure 24. The effect is large when the misalignments are not corrected (as expected) while no notable differences are found between all the equivalent cases studied. This is quantitatively confirmed with the values of the peakto-valley ratio listed in table 2 .

To evaluate the performance of the proposed tool, four users performed the calibration of three simulated situations, described by the misalignments given in table 3. Values were chosen to cover a wide range of possible errors in the worse cases. 

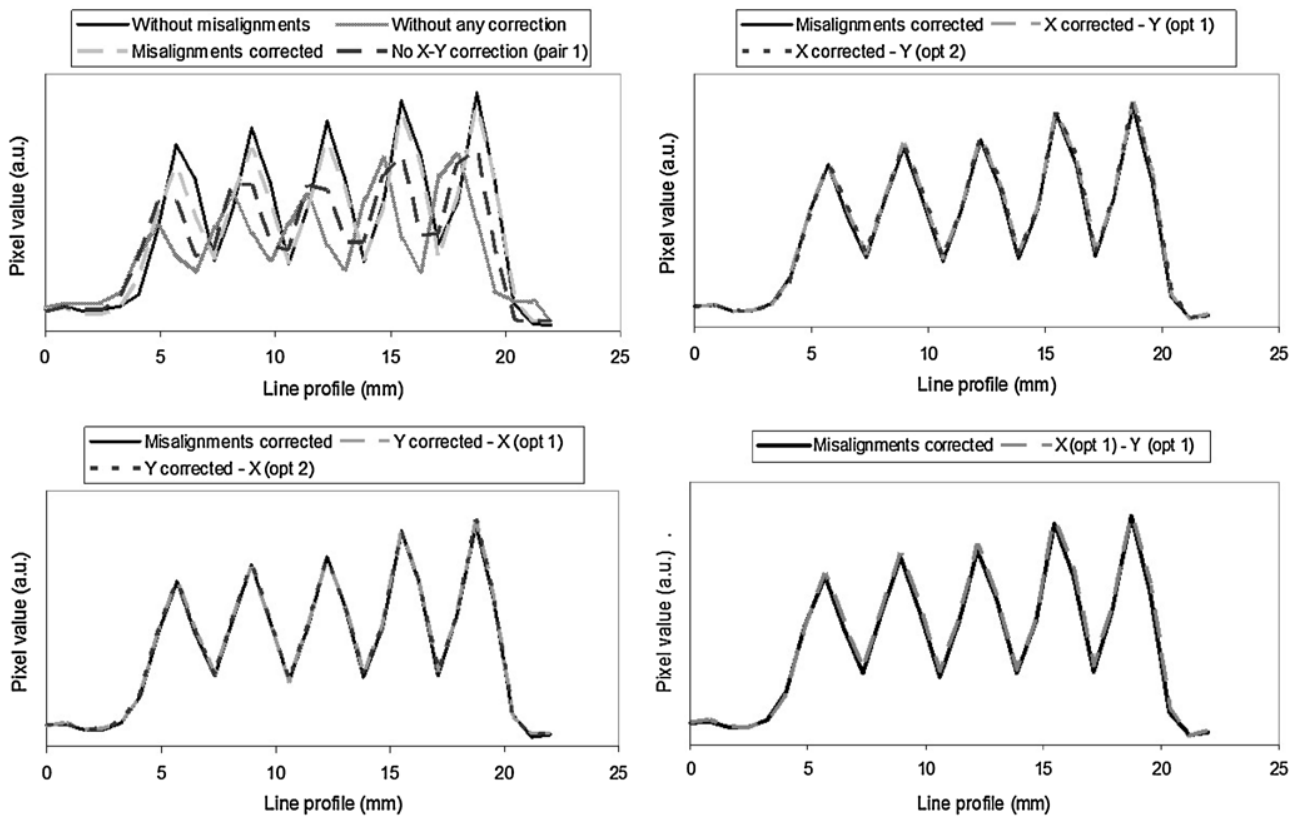

Figure 24. Profiles along a row of rods of $1.6 \mathrm{~mm}$ on the Hot Derenzo Phantom images with different corrections.
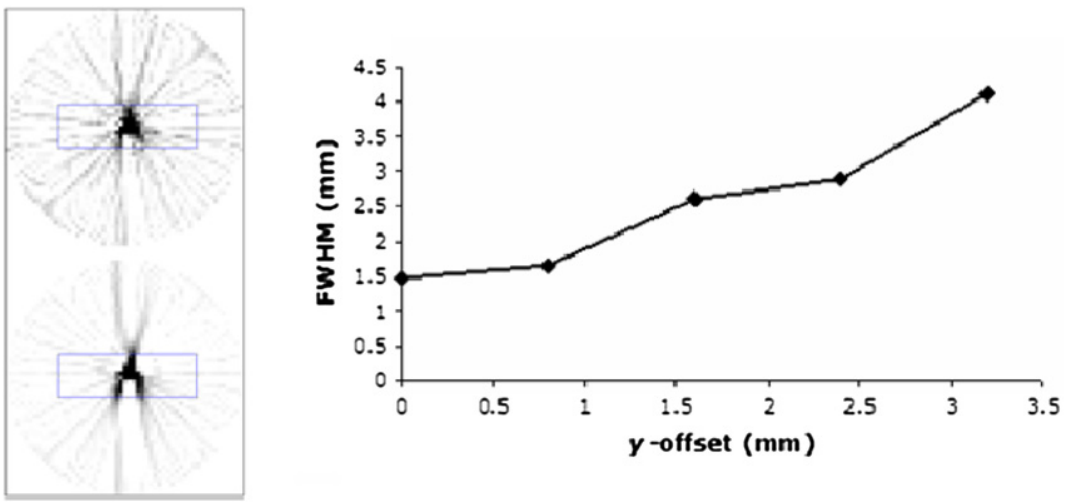

Figure 25. Effect of a $y$-offset on the reconstructed image resolution. Left: transaxial view of a point source reconstruction for a $y$-offset of $0.8 \mathrm{~mm}$ (top) $1.6 \mathrm{~mm}$ (bottom) showing a 'crescent' shape. Right: FWHM of profiles drawn along the $Y_{0}$-axis for different values of the $y$-offset.

The results showed an absolute error within the tolerance value for each parameter when using only one iteration of the algorithm. The complete results are given in table 4.

Finally, we evaluated the impact of the calibration in real studies. Given that the $y$-offset is the one that affects the resolution most within the expected tolerances in a preclinical scanner, we chose this effect for the real cases. Figure 25 shows the effect of a mismatch between the 
Table 3. Misalignment values for the three cases simulated to evaluate the performance of the proposed tool. Each case consists of two studies, one is a non-centered point source and the other one is a point source in the center of the field of view.

\begin{tabular}{|c|c|c|c|}
\hline Misalignment & Case 1 & Case 2 & Case 3 \\
\hline offset_detector1_y (mm) & 1.0 & 0.0 & 0.0 \\
\hline offset_detector2_y (mm) & 0.0 & 2.0 & 1.6 \\
\hline offset_detector3_y (mm) & 0.8 & 1.8 & 0.8 \\
\hline offset_detector4_y (mm) & -0.8 & -0.8 & -0.8 \\
\hline offset_detector1_z(mm) & 0.0 & 0.0 & 0.0 \\
\hline offset_detector2_z (mm) & 1.6 & -2.0 & 0.0 \\
\hline offset_detector3_z(mm) & 3.2 & 3.0 & 0.0 \\
\hline offset_detector4_z(mm) & 1.6 & 0.0 & 0.0 \\
\hline offset_detector1_x (mm) & 0.0 & -10.0 & 0.0 \\
\hline offset_detector2_x (mm) & 7.0 & 0.0 & 5.0 \\
\hline offset_detector3_x (mm) & -3.0 & 5.0 & 2.5 \\
\hline offset_detector4_x (mm) & 5.0 & -5.0 & -2.5 \\
\hline angle_between_detector_pairs (degrees) & 88.0 & 91.0 & 88.0 \\
\hline
\end{tabular}

Table 4. Mean error over the four users for the three coordinates in each case given in table 3 and total mean and standard deviation of the errors.

\begin{tabular}{lllllll}
\hline & \multicolumn{3}{l}{ Mean error over the four users } & Total mean & Total Std. \\
\cline { 2 - 4 } Misalignment & Case 1 & Case 2 & Case 3 & error & Dev. \\
\hline$y$-error (mm) & 0.15 & 0.57 & 0.48 & 0.40 & 0.22 \\
$x$-error (mm) & 0.38 & 0.83 & 2.00 & 1.07 & 1.06 \\
z-error (mm) & 0.00 & 0.90 & 0.00 & 0.30 & 0.49 \\
$\begin{array}{l}\text { angle_between_blocks } \\
\text { (degrees) }\end{array}$ & 0.50 & 0.50 & 0.25 & 0.42 & 0.30 \\
\hline
\end{tabular}

COR and the geometric center on the reconstructed image of a point source: 'crescent' shape of the image that deteriorates resolution. The point source consisting of a sphere $(0.3 \mathrm{~mm}$ of diameter) with $1.1 \mathrm{MBq}(30 \mu \mathrm{Ci})$ of ${ }^{18} \mathrm{Na}$, encapsulated in a $1 \mathrm{~cm}^{3}$ epoxy box, was acquired with the rPET scanner at the center of the FOV in the central slice. Different misalignments were induced by changing the $y$-coordinate in the LOR position when generating the sinograms (over-correction).

Figure 26 illustrates this effect with a rat study affected by a detector misalignment in the range of half the crystal pitch size (one detector was misplaced by $0.8 \mathrm{~mm}$ along the $y$-direction). Acquired data were reconstructed with FBP with a sinogram bow-tie filtering scheme (Abella et al 2009).

\section{Conclusions}

We present a thorough study of the effects of mechanical misalignments in PET scanners based on rotating planar detectors. Our results enable us to propose a robust and easy-to-perform calibration protocol that makes use of simple phantoms to measure misalignments. Although numerous works have analyzed this problem for CT and SPECT systems, information on parallel-beam geometry is scant and only aimed at obtaining the COR. 


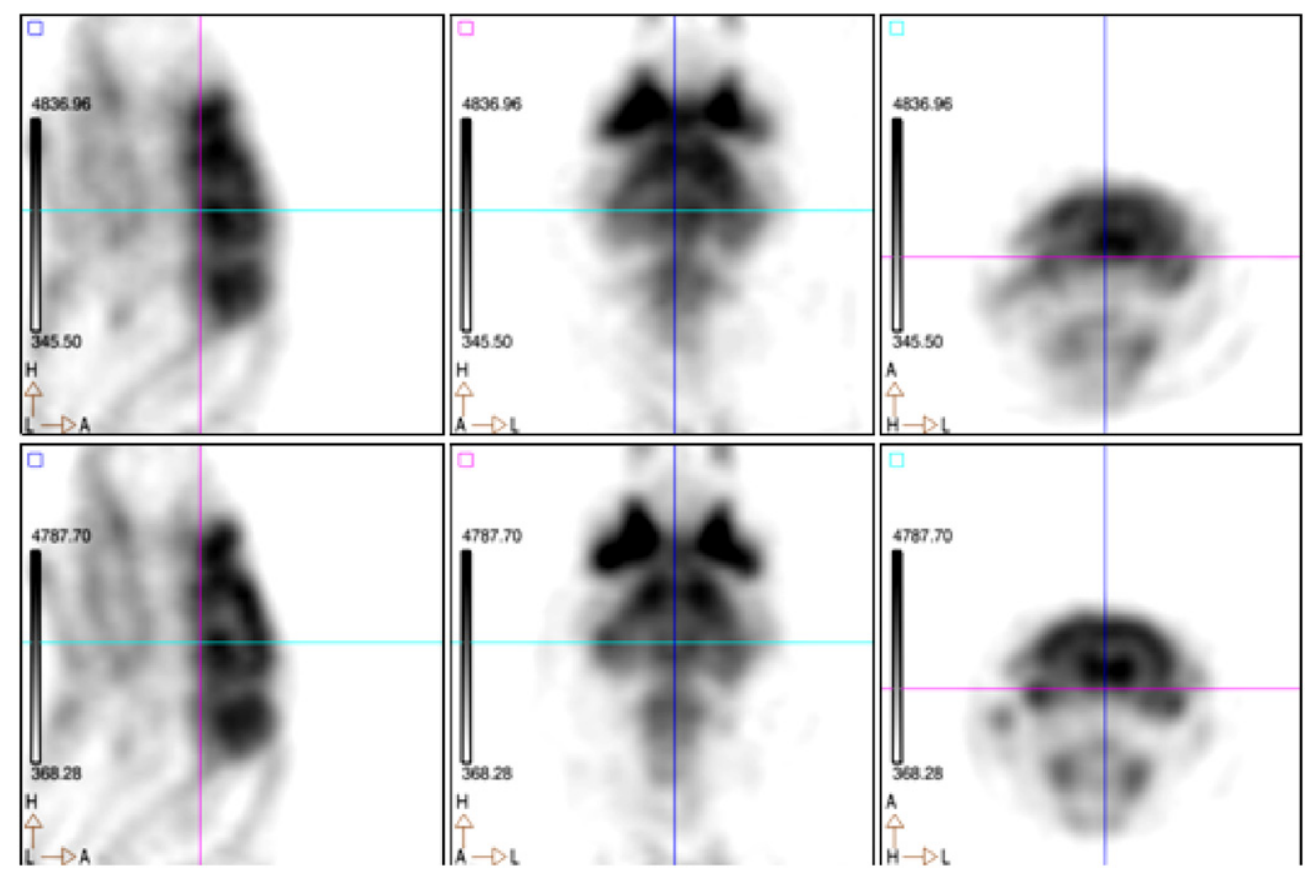

Figure 26. Coronal, sagittal and axial views of an FDG rat study acquired with the rPET scanner and reconstructed with FBP with a bow-tie filtering scheme. Reconstructed volume was $55 \times 55 \times 55$ with $0.8 \mathrm{~mm}$ pixel size. Result before (top) and after (bottom) correcting for misalignments in the range of half the crystal pitch size (one detector was misplaced by $0.8 \mathrm{~mm}$ along the $y$-direction).

We analyzed the effect on reconstructed image quality of the six main misalignment parameters that can exist in a real scanner and conclude that only the linear misalignments along the three axes of the scanner have a relevant effect in practice. This is due to the fact that the minimum tilt errors that would produce any effect in the image are clearly larger than those that would reasonably remain after manufacture. We developed a software tool to generate an offset error file, which can be used during subsequent reconstructions to correct the position of the LORs. Although an iterative reconstruction algorithm could be used, since the calibration process includes several reconstructions, we use FBP to optimize timing. We do not expect to obtain a different misalignment estimation with the proposed method when using an iterative reconstruction since the method is mainly based on sinogram measurements. The evaluation of the proposed calibration tool showed errors within the acceptable tolerance in each geometrical parameter.

The procedure has been incorporated into the software of the commercial rPET system (Vaquero et al 2004, Vaquero et al 2005) and can be easily adapted to similar geometries, as Yap-PET (Del Guerra et al 1998) and ClearPET (Rey et al 2007) scanners. We have demonstrated its versatility in (Lage et al 2009).

Our results highlight the importance of the characterization of detector misalignment: an error of $0.8 \mathrm{~mm}$ in one detector results in an increase of $14 \%$ in the tangential FWHM of a point source at the center of the FOV. Apart from a loss of resolution, misalignment errors also produce artifacts that result in severe degradation of the reconstructed image. 


\section{Acknowledgments}

This work was partially funded by the AMIT project (CEN-20101014) from the CDTICENIT program, RECAVA-RETIC Network (RD09/0077/00087), projects TEC2010-21619C04-01 and TEC2011-28972-C02-01 from the Spanish Ministerio de Ciencia e Innovación, PRECISION grant IPT-300000-2010-3, CPAN (CSD-2007-00042@Ingenio2010), MEC (FPA2010-17142) and ARTEMIS program (S2009/DPI-1802) from the Spanish Comunidad de Madrid and EU-ERDF program. Part of the calculations of this work were performed in the 'Clúster de Cálculo de Alta Capacidad para Técnicas Físicas' funded in part by UCM and in part by UE with european regional funds. This is a contribution for the Moncloa Campus of International Excellence.

\section{References}

Abella M, Vaquero J, Soto-Montenegro M, Lage E and Desco M 2009 Sinogram bow-tie filtering in FBP PET reconstruction Med. Phys. 36 1663-71

Abella M, Vaquero J J, Vicente E, Álvarez J, Lage E and Desco M 2006 Effect of misalignments in small animal PET scanners based on rotating planar detectors Mol. Imaging Biol. 8 75-6

Azevedo S G, Schnebeck D J, Fitch J P and Martz HE 1990 Calculation of the rotational centers in computed tomography sinograms IEEE Trans. Nucl. Sci. 37 1525-40

Baró J, Sempau J, Fernández-Varea J M and Salvat F 1995 PENELOPE: An algorithm for Monte Carlo simulation of the penetration and energy loss of electrons and positrons in matter Nucl. Instrum. Methods Phys. Res. B $10031-46$

Beque D, Nuyts J, Bormans G, Suetens P and Dupont P 2003 Characterization of pinhole SPECT acquisition geometry IEEE Trans. Med. Imaging 22 599-612

Busemann-Sokole E 1987 Measurement of collimator hole angulation and camera head tilt for slant and parallel-hole collimators used in SPECT J. Nucl. Med. 28 1592-8 PMID: 3498806

Del Guerra A, Di Domenico G, Scandola M and Zavattini G 1998 YAP-PET: first results of a small animal positron emission tomograph based on YAP:Ce finger crystals IEEE Trans. Nucl. Sci. 45 3105-8

Donath T, Beckmann F and Schreyer A 2006 Automated determination of the center of rotation in tomography data J. Opt. Soc. Am. 23 1048-57

España S, Herraiz J L, Vicente E, Vaquero J J, Desco M and Udias J M 2009 PeneloPET, a Monte Carlo PET simulation tool based on PENELOPE: features and validation Phys. Med. Biol. 54 1723-42

Gullberg G T, Tsui B M W, Crawford C R, Ballard J G and Hagius J T 1990 Estimation of geometrical parameters and collimator evaluation for cone beam tomography Med. Phys. 17 264-72

Gullberg G T, Tsui B M W, Crawford C R and Edgerton E 1987 Estimation of geometrical parameters for fan-beam tomography Phys. Med. Biol. 32 1581-94

Hsieh Y-L 1992 Calibration of Fan-Beam Geometry for Single Photon Emission Computed Tomography MS Thesis University of Utah, Salt Late City

Jagoda E M, Vaquero J J, Seidel J, Green M V and Eckelman W C 2004 Experiment assessment of mass effects in the rat: implications for small animal PET imaging Nucl. Med. Biol. 31 771-9

Lage E, Vaquero J J, Sisniega A, Espana S, Tapias G, Abella M, Rodriguez-Ruano A, Ortuno J E, Udias A and Desco M 2009 Design and performance evaluation of a coplanar multimodality scanner for rodent imaging Phys. Med. Biol. 54 5427-41

Lecomte R 2004 Technology challenges in small animal PET imaging Nucl. Instrum. Methods Phys. Res. A 527 157-65

Lewis J S and Achilefu S 2002 Small animal imaging: current technology and perspectives for oncological imaging Eur. J. Cancer 38 2173-88

Massoud T F and Gambhir S S 2003 Molecular imaging in living subjects: seeing fundamental biological processes in a new light Genet. Dev. 17 545-80

Noo F, Clackdoyle R, Mennessier C, White T A and Roney T J 2000 An analytic method based on identification of ellipse parameters for scanner calibration in conebeam tomography Phys. Med. Biol. 45 3489-508

Pierce L, Miyaoka R, Lewellen T, Alessio A and Kinahan P 2009 Determining block detector positions for PET scanners IEEE Nucl. Sci. Symp. Conf. Record pp 2976-80

Pomper M G 2001 Molecular imaging: and overview Acad. Radiol. 8 1141-53 
Rey M, Jan S, Vieira J M, Mosset J B, Krieguer M, Comtat C, Morel C and Dahlbom M 2007 Count rate performance study of the Lausanne ClearPET scanner demonstrator Nucl. Instrum. Methods Phys. Res. A 571 207-10

Sun Y, Hou Y and Hu J 2007 Reduction of artifacts induced by misaligned geometry in cone-beam CT IEEE Trans. Bio. Eng. 54 1461-71

Vaquero J J, Lage E, Ricón L, Abella M, Vicente E and Desco M 2005 rPET detectors design and data processing IEEE Nuclear Science Symp. Conf. Rec. 5 2885-9

Vaquero J J, Molins A, Ortuño J, Pascau J and Desco M 2004 Preliminary results of the small animal rotational positron emission tomography scanner Mol. Imaging Biol. 6102 